\title{
MS-Based Analytical Techniques: Advances in Spray-Based Methods and EI-LC-MS Applications
}

\author{
Federica Bianchi $\left(\mathbb{D},{ }^{1}\right.$ Nicolò Riboni $\left(\mathbb{D},{ }^{1,2}\right.$ Veronica Termopoli $\mathbb{D}^{3},{ }^{3}$ Lucia Mendez ${ }^{(D},{ }^{4}$ \\ Isabel Medina, ${ }^{4}$ Leopold Ilag $\left(\mathbb{D},{ }^{2}\right.$ Achille Cappiello, ${ }^{3}$ and Maria Careri ${ }^{1}$ \\ ${ }^{1}$ Department of Chemistry, Life Sciences, and Environmental Sustainability, University of Parma, \\ Parco Area delle Scienze 17/A, 43124 Parma, Italy \\ ${ }^{2}$ Department of Environmental Science and Analytical Chemistry, Stockholm University, 10691 Stockholm, Sweden \\ ${ }^{3}$ Department of Pure and Applied Sciences, LC-MS Laboratory, Piazza Rinascimento 6, 61029 Urbino, Italy \\ ${ }^{4}$ Instituto de Investigaciones Marinas, Spanish National Research Council (IIM-CSIC), Eduardo Cabello 6, 36208 Vigo, Spain \\ Correspondence should be addressed to Federica Bianchi; federica.bianchi@unipr.it
}

Received 22 December 2017; Accepted 26 February 2018; Published 23 April 2018

Academic Editor: Gauthier Eppe

Copyright (c) 2018 Federica Bianchi et al. This is an open access article distributed under the Creative Commons Attribution License, which permits unrestricted use, distribution, and reproduction in any medium, provided the original work is properly cited.

\begin{abstract}
Mass spectrometry is the most powerful technique for the detection and identification of organic compounds. It can provide molecular weight information and a wealth of structural details that give a unique fingerprint for each analyte. Due to these characteristics, mass spectrometry-based analytical methods are showing an increasing interest in the scientific community, especially in food safety, environmental, and forensic investigation areas where the simultaneous detection of targeted and nontargeted compounds represents a key factor. In addition, safety risks can be identified at the early stage through online and real-time analytical methodologies. In this context, several efforts have been made to achieve analytical instrumentation able to perform real-time analysis in the native environment of samples and to generate highly informative spectra. This review article provides a survey of some instrumental innovations and their applications with particular attention to spray-based MS methods and food analysis issues. The survey will attempt to cover the state of the art from 2012 up to 2017.
\end{abstract}

\section{Introduction}

Mass spectrometry (MS) is one of the most powerful techniques for the detection and identification of organic and inorganic compounds. Being able to provide both molecular weight and structural information [1], it is widely used in analytical laboratories for academic research, industrial product development, and regulatory compliance as well as for proteomic or metabolomic studies, DNA characterization, drug discovery, environmental monitoring, food analysis, forensics, and homeland security.

A plethora of analytical MS-based methods based on the use of both stand-alone instruments and mass spectrometers coupled to different separation techniques such as gas and liquid chromatography (GC and LC) or capillary electrophoresis (CE) have been developed and validated in order to analyze complex matrices. Interesting review articles and book chapters dealing with advances in ionization for mass spectrometry have been lately published [2-9].

Recently, the advent of ambient MS technology paved the way for the development of a great variety of applications and innovations characterized by high throughput: the challenge of analyzing samples in their native state without sample treatment encouraged the development of new techniques among which are the spray-based ionization ones including desorption electrospray ionization (DESI) [10], paper spray ionization (PSI) [11], laser ablation electrospray ionization (LAESI) [12], and easy ambient sonic-spray ionization (EASI) [13].

Novel materials and new instrumental configurations are under study to enhance the performance of the different ion sources. Safety risks can be identified at the early stages 
through nontargeted monitoring technologies. Furthermore, the variety of fragmentation strategies that can be combined in new instrumentation overall enhances work in the omics fields, particularly proteomics and metabolomics.

Although MS-based methods are getting progressively more powerful, reliable, and easily available, the main drawbacks are still related to sample complexity and preparation, mass accuracy, often requiring the use of highresolution mass spectrometry (HRMS) to guarantee the univocal identification of the targeted compounds, and the need of high-throughput and screening analyses when a great number of samples have to be analyzed.

The aim of the proposed special issue is to cover the aspects regarding emerging features of MS-based techniques focusing on innovative LC-MS studies and ambient MS with particular attention to the spray-based ionization techniques. New materials, prototypes, and instrumental configurations able to increase the performance of the developed methods will be presented and discussed. Finally, an overview of the most recent MS-based methods in food analysis will be given. This survey will attempt to cover the state of the art from 2012 up to 2017.

\section{Advances in LC-MS}

Electrospray ionization (ESI) is the technique of choice to produce ions suitable for mass analysis. ESI spectra typically are characterized by single protonated or deprotonated molecular ion $(\mathrm{M}+\mathrm{H})^{+},(\mathrm{M}-\mathrm{H})^{-}$, and/or adduct ions. The low fragmentation is a limitation in compound characterization through the use of reliable electronic libraries, making necessary the use of multistage MS (MS/MS, $\mathrm{MS}^{n}$ ) or HRMS to compensate the limited structural information. LC-ESI with triple quadrupole (QqQ) MS is the most used technique for qualitative and quantitative determination of targeted nonvolatile compounds in forensic and food applications $[14,15]$. In 2016, Remane et al. reviewed the literature on applications of LC-MS/MS in clinical, forensic toxicology, and doping control since 2006 [16]. It must be noted that ESI response is strictly affected by the mobile phase and sample composition as well as by the presence of coeluting interfering compounds, which may interfere with the ionization process. These phenomena are known as "matrix effects" (MEs) and can alter the response of the analytes causing either signal suppression or enhancement $[17,18]$. The occurrence of ME introduces some critical analytical shortcomings in quantitative analysis by LC-MS such as reduced sensitivity, nonlinear response, and low precision. In addition, the physicochemical properties of the analytes can play an issue in the instrument response, thus introducing additional limitations. The combination of powerful MS detectors with LC has solved many problems in structural elucidation of unknown hazardous compounds [19]. In this context, HRMS is capable of providing full spectral information by adding high mass resolving power and accuracy to achieve selectivity and capability for accurate mass measurements [20-22]. HRMS is characterized by higher mass resolution, defined as the mass difference between two mass spectral peaks that can be clearly distinguished [23], and higher mass accuracy (even better than $1 \mathrm{ppm}$ ). In addition, high mass resolving power allows discrimination between isobaric interference and ion of interest, leading to an accurate mass measurement even with a complex background. These features increase MS selectivity for the screening of nontargeted compounds in complex matrices, providing a list of possible elemental compositions. Fu et al. have shown how important and efficient is the use of nontargeted screening with LC-HRMS to ensure quality and safety of food [24], whereas Mattarozzi and coworkers exploited the capability of HRMS for the rapid determination of melamine from melamine tableware [25]. On the downside, HRMS-based methods generate complicated data that must be processed for "total ion fragment spectra" to obtain high-quality mass spectral information. Moreover, the mass of protonated or deprotonated molecules is not sufficient to prevent unambiguous compound identification. Hence, the use of spectramatching approaches that utilize fragmentation ions could be added to achieve additional information on the detected compounds [26, 27]. False positives and false negatives are the major obstacles when screening complex samples. False negatives can occur due to very low concentrations, matrix interferences and suppression, and weak or no ionization. Due to these disadvantages, the practical application of LCMS and LC-HRMS is still far from the immediacy and simplicity of GC-MS. Taking into account that electron ionization (EI) allows us to obtain characteristic and highly reproducible fragmentation of the analytes, a considerable effort has been devoted by the scientific community to increase compatibility between LC and EI-based MS to develop reliable, easy-to-use, and flawless interfaces. Moreover, implementation of EI fragmentation to LC-amenable compounds could pave the way for many new fields of research.

Recent developments in miniaturized mass spectrometers have enabled these developments to be carried out to portable on-scene detection. In the next paragraphs, some of the most popular and promising techniques are described: among them are LC-MS based on EI interfaces and spraybased ionization techniques.

2.1. LC-MS Based on Electron Ionization-Mass Spectrometry Interfaces. It is known that EI is ideal for the detection of a large number of GC-amenable compounds, but with an appropriate combination of several measures, it can become suitable for many LC-amenable small molecules having MW up to approx. $600 \mathrm{Da}$. These compounds can be efficiently converted into the gas phase, fast enough to be ionized before any decomposition process.

For analysis of small-to-medium molecules, the coupling between LC and EI-MS represents a valid strategy for overcoming the main disadvantages related to ESI ionization and the use of costly and complicated techniques involving HRMS instrumentations. Furthermore, EI provides a rich fragmentation pattern with a significant amount of structural information allowing a unique automated identification with structures at the isomer level [28]. Hence, the 
ability of EI for tentative identification of GC-amenable compounds is unparalleled even without HRMS.

On the downside, the coupling between LC and MS based on EI represents a significant issue in the field of analytical chemistry. The reason may be explained by the antagonist conditions of operating. The first one typically works at ambient temperature and uses very high pressure for the efficient separation of the analytes, which are sometimes dissolved in a complex mobile phase. The second one operates at a very high vacuum and high temperature. Therefore, the effort of achieving and maintaining the high vacuum required for mass spectrometry is in contrast with the intrinsic nature of HPLC, predominantly operating at high solvent flow rates. Also, the low tolerance of mass spectrometers for nonvolatile mobile phase components contrasts with an HPLC dependence on nonvolatile buffers to achieve high-resolution separations.

Since the year 2000, a few groups of researchers are working on the development of an efficient EI-based LC-MS interface.

Cappiello and his group played a significant role in the innovation and improvement of LC-EI-MS interfacing and designing a series of systems characterized by steadily increasing performance. Firstly, they presented a prototype of the LC-MS interface called Direct-EI [29-31] based on direct coupling of a low flow rate nano-HPLC with a high-vacuum EI source. The interface governs the direct introduction of a liquid-phase sample into the EI source of the mass spectrometer and the complete conversion of the liquid effluent to the gas phase prior to a conventional electronassisted ionization. The core of the interface is represented by the nebulizer, which consists of a fused silica/PEEK capillary, to guarantee a sufficient thermal insulation. This interface was used in many different applications, not only in combination with chromatography but also in direct analysis, as a universal detector for the targeted compound. However, both nebulization and vaporization take place inside the ion source, leading to some drawbacks linked to capillary blockings. This concern is mainly due to premature evaporation of the solvent making the analysis very difficult under routine conditions. To meet the challenges of analyzing nontargeted compounds exploiting full potential of EI and the quantification of target compounds at low concentration in complex matrices, Termopoli et al. presented a new, robust, efficient interfacing mechanism coming from the ground up [32]. The new interface is called "liquid-EI" (LEI). The interface is completely independent from the rest of the instrumentation and can be adapted to any gas chromatography-mass spectrometry system, as an add-on for a rapid LC-MS conversion. Secondly, with some little tricks, it can be used with any HPLC system. Nanopumps and capillary pumps allow direct connection, and conventional HPLC needs the use of a two-way splitter to reduce the column flow rate to a level that is compatible with the interface, which is normally between 0.5 and $1 \mu \mathrm{l} / \mathrm{min}$. In an LEI interface, the vaporization of the LC eluate is carried out at atmospheric pressure inside a suitable, independent microchannel right before entering the ion source, called the vaporization microchannel, representing the core of the interface. It is designed to uncouple and separate the atmospheric pressure found at the end of the HPLC system with the high-vacuum zone of the ion source. This specific place is narrow enough to prevent vacuum from entering into the spray region allowing us to have an atmospheric pressure zone where the vaporization process takes place. A removable silica-deactivated liner ensures a perfect conversion into a gas phase before entering the mass spectrometer. A narrower fused silica capillary, called the inlet, penetrates in the first portion of the liner and releases the LC eluate. An inert gas flow surrounds the gas phase through the vaporization microchannel and helps high boiling compounds to vaporize. Figure 1 shows a complete layout of the LC-MS system equipped with the LEI interface.

The rapid vaporization offered by the lined microchannel reduces the chance of thermal decomposition and capillary blockings, broadening the range of suitable applications, especially those regarding nontargeted analytes. Remarkable results were achieved in different conditions and applications.

Over the past years, Seemann and his group developed the supersonic molecular beams (SMB) LC-MS interface [34]. Their studies started from the knowledge that standard emission energy $(70 \mathrm{eV})$ used in EI is not ideal for many NIST library compounds that have a weak (below $2 \%$ relative abundance) or no molecular ion. This issue is a critical point when very large and thermally labile compounds are analyzed. Furthermore, these analytes are usually less volatile and require higher EI ion source temperatures with further intra-ion source degradation, resulting in weaker molecular ion production. To achieve a reliable EI-based sample identification, a more intense production of molecular ion is needed. Thus, the best ionization method should provide the informative library searchable EI fragments combined with enhanced molecular ions, especially for the compounds that are not included in the commercially available EI libraries. Taking into account these considerations, they present a novel concept of the LC-SMB-MS system, based on the use of supersonic molecular beams, as a medium for electron ionization of vibrationally cold sample molecules in a flythrough ion source. It is able to generate library searchable EI spectra and a more intense molecular ion.

The LC-SMB-MS apparatus is schematically shown in Figure 2.

A thorough evaluation of the interface, comprising identification of unknown compounds using obtained library searchable EI mass spectra, enhanced production of molecular ions, demonstration of the absence of matrix effects, simultaneous determination of semipolar and nonpolar compounds with reasonable detection limit, and low-cost instrumentation, was provided by that research group. The group of Seemann demonstrates the feasibility of the SMB interface as a valid tool in the analysis of unknown compounds and as a low-cost LC-EI-MS system.

A third group of researchers, headed by Rigano, presented a new nano-LC-EI-MS for the determination of free fatty acids (FFAs) in mussels [34]. A selective and sensitive nano-LC-EI-MS analytical method to investigate the FFA profile in marine organisms and to monitor marine sentinels 

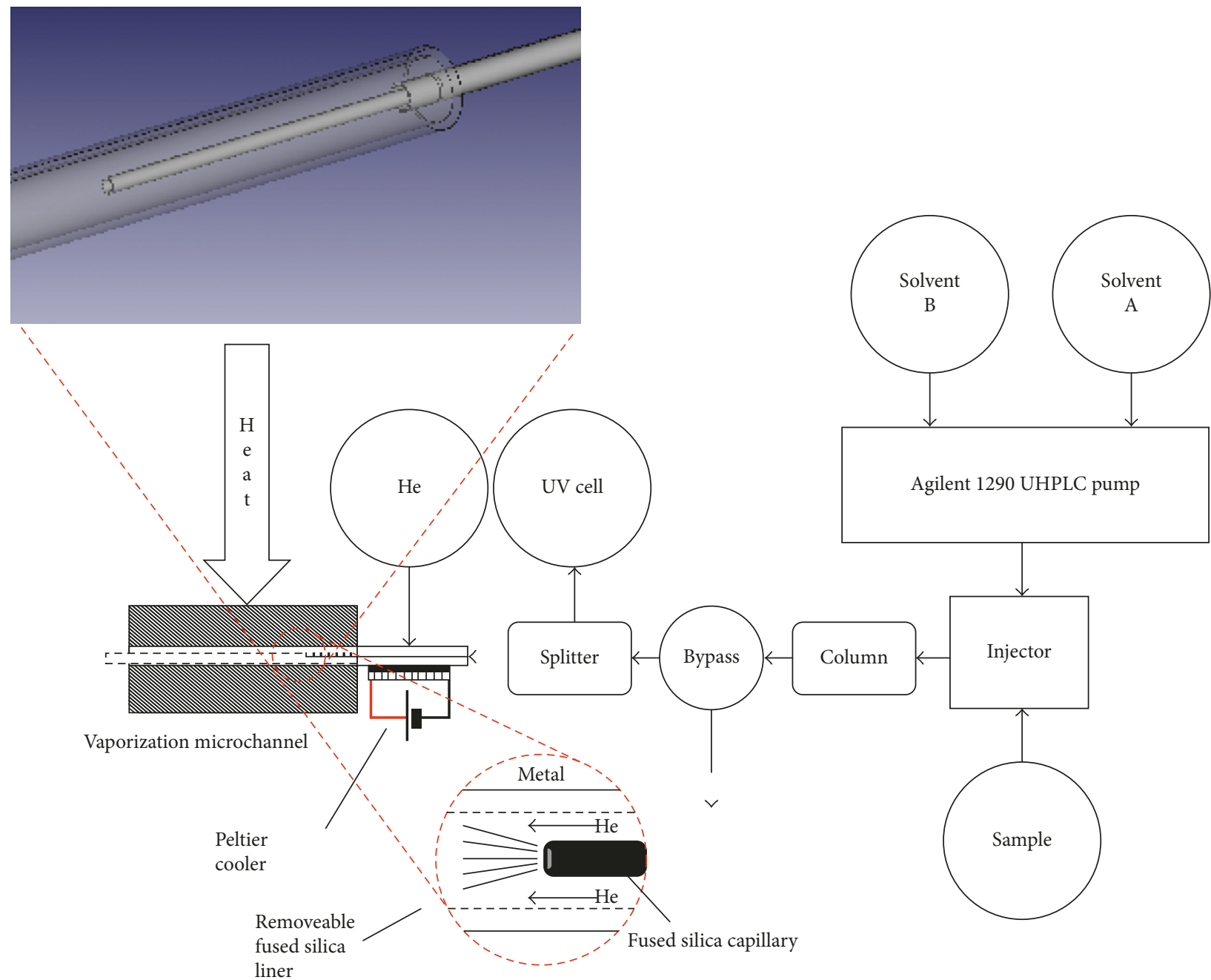

Agilent 1290 UHPLC pump

FIGURE 1: Global layout of the fully assembled system; the LEI interface, in gray, is between the UHPLC system and the MS detector. In the red circle, the vaporization zone is highlighted. Reprinted with permission from [33].

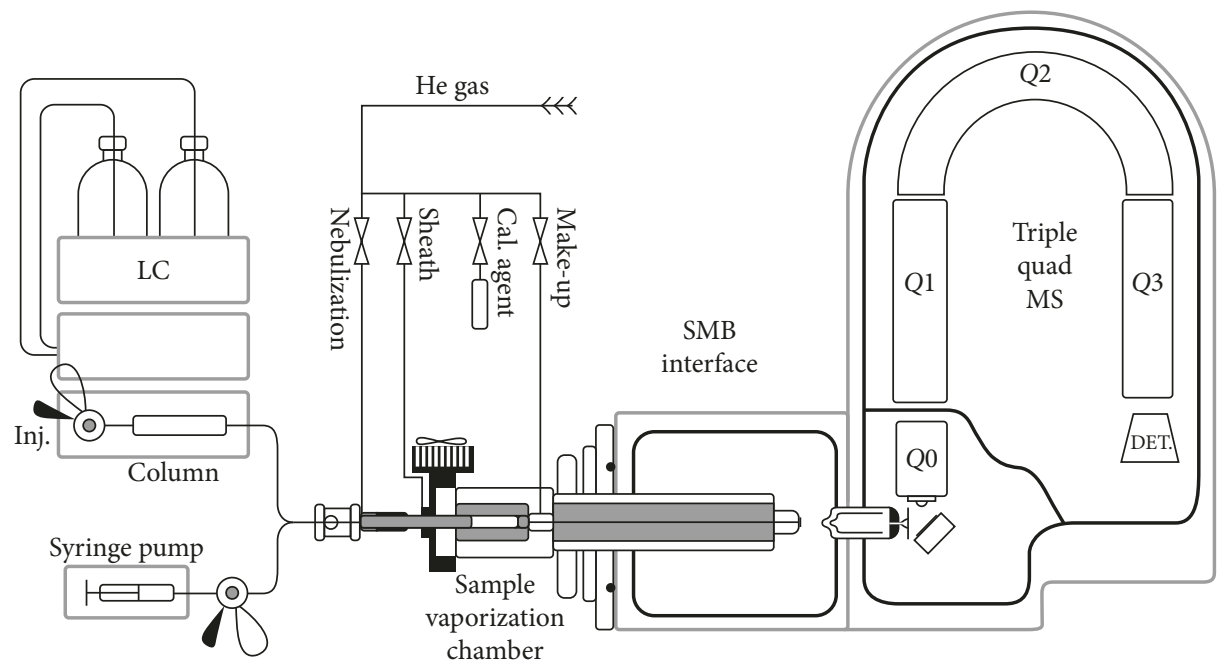

Figure 2: EI-LC-MS with the SMB system outline. The liquid is introduced either from the HPLC system after its column or from a syringe pump to the heated vaporization chamber through a pneumatic nebulizer. The helium nebulization gas enters the SMB interface through a nebulization gas line, sheath gas line, and nozzle make-up gas line. Reprinted with permission from [35]. 
for the assessment of environmental pollution effects was developed [35]. FFAs are minor components of the lipidome, and they are usually analyzed by GC after a derivatization step, such as methylation or trimethylsilylation, is performed to convert FA into less-polar and more-volatile moieties and improve their separation $[9,36]$. However, the derivatization step, if not properly selected, can modify the FA profile due to nonhomogeneous derivatization efficiency among different compounds (saturated, unsaturated, and polyunsaturated fatty acids). In addition, oxidation or isomerization products can be generated. Relative to this issue, LC can benefit over GC techniques from direct injection of FFAs in their intact form, without any pretreatment. On the other hand, direct coupling with EI-MS can benefit from the highly informative, repeatable, and reliable MS fragmentation. Drawing conclusions of the several attempts made by each group, they are following a distinctive pathway to obtain a common goal, the development of a more useful and universal LC-EI-MS interface.

Regarding Direct-EI LC-MS, recent studies have been carried out also to increase the inertness of the electron ionization ion source by developing new materials [37, 38].

As already stated, the vaporization surface of an electron ionization MS source is a key parameter for the detection and characterization of targeted and untargeted analytes: it is known that difficulties in the vaporization process arise when compounds characterized by high molecular weight and/or polarity have to be analyzed, thus requiring both the use of inert ion sources to reduce the interactions of the analytes with the stainless steel ion source and the use of high source temperatures to promote analyte vaporization. In this field, Magrini et al. [37] proved that the use of a commercially available ceramic coating is able to improve the detection of high molecular weight and high boiling compounds like polycyclic aromatic hydrocarbons (PAHs) and hormones. More recently, Riboni et al. [38] were able to increase the inertness of the electron ionization ion source by developing different sol-gel coatings based on silica, titania, and zirconia. Again, the developed coatings were tested for the Direct-EI LC-MS determination of PAHs and steroids. The best performances in terms of both signal peak intensity and peak width were obtained by using the silicabased coating, obtaining detection limits in the low $\mathrm{ng} / \mathrm{ml}$ range with a good precision (RSD $<9 \%$ for PAHs and $<11 \%$ for hormones). No problems associated to ion cleaning were observed after prolonged use.

\section{Advances in Spray-Based Ionization Techniques}

Nowadays, there is also a growing interest in the development of real-time analytical technologies capable of allowing the direct detection of trace analytes in complex samples, especially in their native environment. The development of a new class of techniques, better known as "ambient ionization techniques," has introduced a revolution in the ionization field. These techniques are able to generate ions directly from native environment of the sample at ambient pressure, without any tedious sample preparation steps or laborious time-consuming chromatographic separation.

Technically, spray-based ionization techniques are based on the use of electrospray droplets to extract the analytes from the sample and transfer them to the mass spectrometer. The most common spray-based ionization technique is DESI, in which a high-velocity pneumatically assisted ESI source generates charged microdroplets by the application of a proper potential on the ESI needle. The spray is directed towards the sample where the impact of the primary droplets with the substrate leads to the formation of a micrometersized thin solvent film, able to solubilize the analytes at the liquid-solid interface. Secondary droplets containing the analytes expelled by the film solvent are generated, and then, desolvation and ionization in the gas phase occur, as in the traditional ESI analysis. Finally, the ions are collected by the MS inlet.

In addition to DESI, several other techniques like nanoDESI, EASI, and LAESI have been proposed. Probe electrospray ionization (PESI) is another interesting approach based on the use of a solid conductive needle probe that replaces the traditional electrospray capillary for sample introduction. Similarly, PSI is a technique based on the loading of the sample onto a triangular piece of paper from which ions are generated by applying a high voltage in the presence of a proper solvent [11]. Spray-based methods are suitable for the analysis of different compounds, from small analytes, such as explosives [39-43], drugs [44-47], and food contaminants [48-50], to larger molecules such as lipids [51-53], peptides $[54,55]$, and proteins $[56,57]$.

3.1. Desorption ElectrosprayIonization-Mass Spectrometry. DESIMS is usually applied for surface desorption/ionization of analytes deposited on a probe material (PTFE, PMMA, glass, etc.) or directly from the sample surface. DESI-MS and DESI imaging have been successfully applied in different fields, such as forensic science [58], food control [59, 60], and clinical applications [61-63].

The derivatization of metabolites deposited in solution onto a glass plate by dropping the derivatizing reagent on the top of the dried analytes was proposed by Lubin et al. [64]. The authors successfully applied this technique to several samples, demonstrating the possibility of performing multiple and subsequent derivatization steps on the same spot.

An interesting approach was developed by Brown et al. [65] for the MS detection of fleeting reaction intermediates in electrochemical reactions utilizing a new waterwheel working electrode setup. The proposed technique allowed us to exploit DESI-MS operating at a low voltage. The new apparatus consisted of a round rotating platinum working electrode that was partially immersed in an aqueous electrolyte solution (Figure 3). During the rotation, a thin layer of liquid film was deposited on the electrode surface, as in a waterwheel. A three-electrode system was set by using a platinum wire counterelectrode and an $\mathrm{Ag} / \mathrm{AgCl}$ reference electrode, immersed in the reservoir of electrolyte solution. The upper surface of the waterwheel was hit by a spray generated by a custom spray probe, thus allowing the 


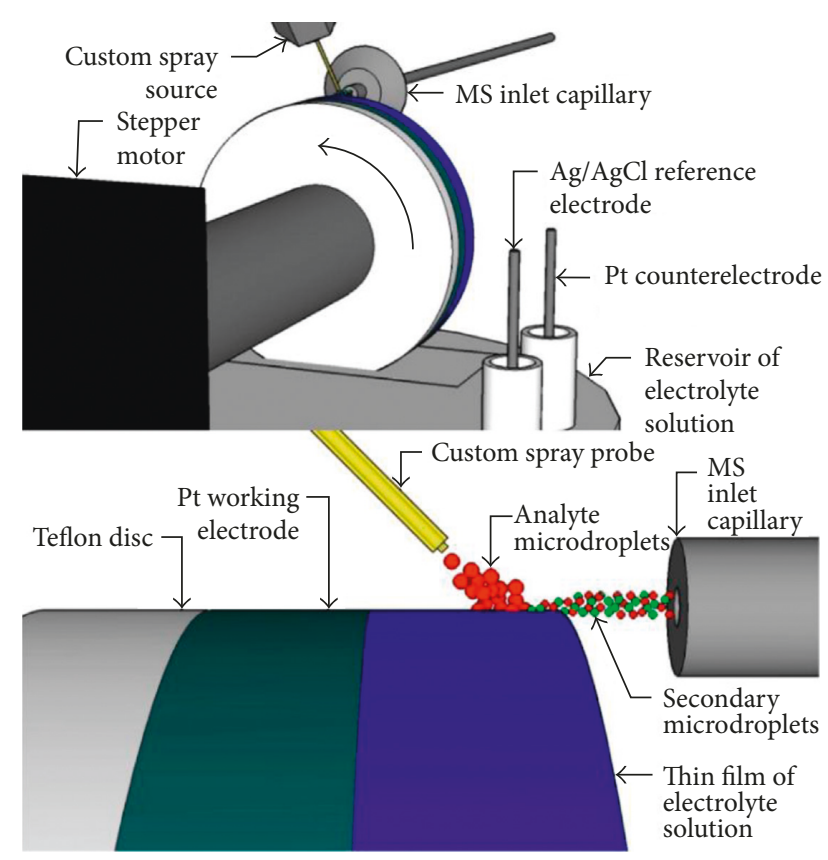

FIGURE 3: Schematic representation of the developed experimental setup. Reprinted with permission from [65].

formation of secondary droplets, analyte ionization, and their collection in the MS inlet. To avoid any electrochemical oxidation or reduction on the electrode surface, no high voltage was applied to the analyte spray, whereas a low potential (few volts) was applied to the platinum rotating electrode.

The authors tested the new apparatus towards the detection of a diimine intermediates during electrochemical oxidation of both uric acid and xanthine.

An MS-electrochemistry coupling was also proposed by Looi et al. [66], who developed a new online electrochemistryliquid sample desorption electrospray ionization-mass spectrometry (EC-LS DESI-MS) system. In EC-LS DESI-MS, an electrosonic spray ionization source was used to generate a spray directed to the exit of the liquid sample capillary positioned perpendicularly to the spray and the MS inlet. Separately, a thin two-electrode flow-through EC cell was connected to a syringe pump and was used to perform oxidation/reduction processes. The ESSI-generated spray was able to impact the outer surface of the LS capillary, which is continuously coated by sample solution flowing at $200 \mu \mathrm{l} / \mathrm{h}$, thus allowing the ionization of the analytes. This prototype was developed and tested using $N, N$-dimethyl-p-phenylenediamine (DMPA). Although oxidation of DMPA was already observed as a result of ionization of DESI-MS in positive mode, by applying a proper voltage to the online electrochemical (EC) cell, it was possible to increase the yields of the oxidation products, thus improving method sensitivity.

Although DESI is usually coupled with high-resolution mass spectrometry, its coupling with LC is possible. A novel splitting method for LC-MS applications, which allows both very fast MS detection of analytes eluted from the LC column and their online collection, was presented by Cai et al.
$[67,68]$. In this approach, a PEEK capillary tube with a micro-orifice is used to couple DESI with the UPLC column. By using the proposed instrumental setup, a small amount of LC eluent (few nanoliters) is ionized by DESI with negligible time delay $(6 \sim 10 \mathrm{~ms})$, whereas the remaining analytes exiting the tube outlet can be collected. In addition, online derivatization using reactive DESI is feasible increasing the charge of proteins and consequently enhancing the ionization yields.

An interesting novel configuration has been recently developed by Ren et al. [69]. The authors developed a method coupling slug-flow microextraction (SFME) and nanoelectrospray ionization for the MS analysis of organic compounds in blood and urine. A disposable glass capillary with a pulled tip for nano-ESI was used to perform the entire extraction and ionization process (Figure 4). Two adjacent liquid plugs were formed by injecting $5 \mu \mathrm{l}$ of a proper organic solvent and $5 \mu \mathrm{l}$ of body fluid (urine or blood) into the capillary. Liquid-liquid extraction of the analytes was performed by both moving the capillary and applying a pushand-pull force through air pressure. After the extraction process, a high voltage is applied to the organic solvent plug to generate the nano-ESI for MS analysis.

The proposed method was tested for the extraction and detection of different analytes, namely, methamphetamine, benzoylecgonine, verapamil, amitriptyline, epitestosterone, 6-dehydrocholesterone, $5 \alpha$-androstane- $3 \beta, 17 \beta$-diol-16-one, and stigmastadienone. Major analytical features were the reduced consumption of both the organic solvent and sample. The authors demonstrated that a direct derivatization of the extracted analytes in the organic phase was feasible, thus achieving excellent sensitivity with detection limits in the $0.03-0.8 \mathrm{ng} / \mathrm{ml}$ range.

Despite its name, nanospray DESI (nano-DESI) is based on a different instrumental configuration compared to the traditional DESI: its setup presents two different silica capillaries, one for solvent delivery and the other devoted to the formation of charged liquid spray in front of the MS inlet. The two capillaries are not in direct contact, thus producing a solvent bridge on the DESI surface. The second nanospray capillary produces a self-aspirating nanospray, which is generated by applying a high voltage between the MS inlet and the primary capillary. In comparison with DESI, nano-DESI is characterized by higher efficiency in liquid transportation and sampling performances.

The capabilities of nano-DESI-MS were tested for the determination of pollutants and organic components in atmospheric fine particles by Cain et al. [70], in environmental aerosol by Tao et al. [71], and in clouds by Boone et al. [72]. In the clinical and pharmaceutical fields, both nanoDESI-MS and nano-DESI-MS imaging proved to be excellent techniques for the analysis of pharmaceuticals, biomolecules, and metabolites [73-77].

A further instrumental innovation has been proposed by Duncan and coauthors [78], who developed a pneumatically assisted nanospray desorption electrospray ionization source. The instrumental setup was based on the introduction of a secondary nebulizer replacing the selfaspirating secondary capillary in order to assist solvent 


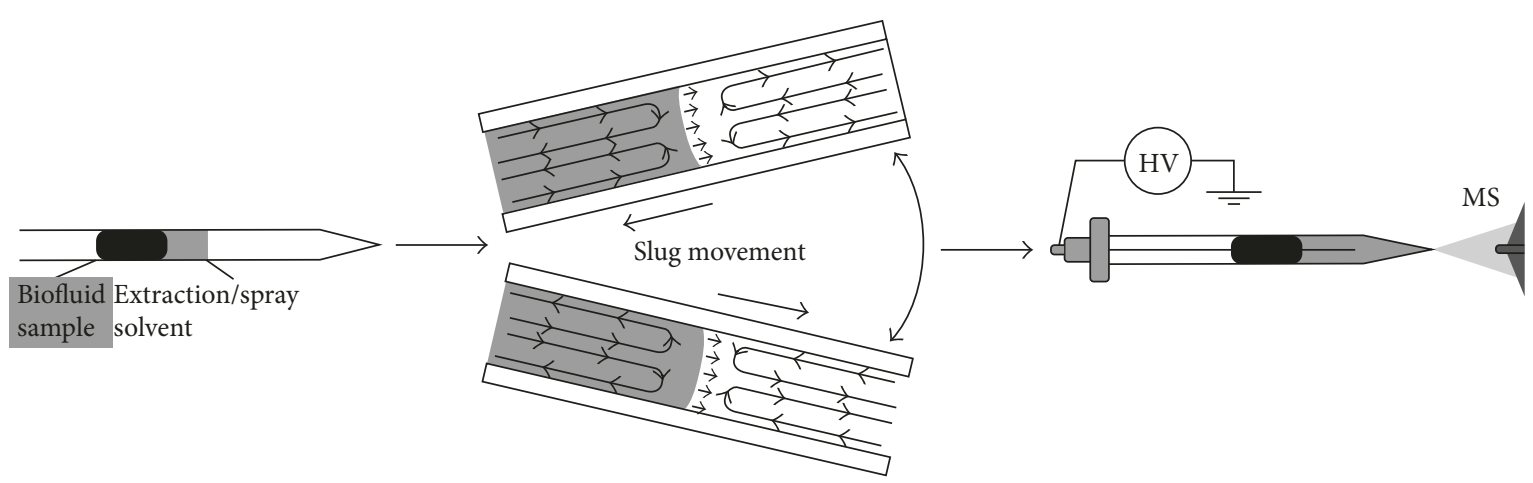

FIgURE 4: Schematic representation of the SFME-nano-ESI sample processing. Reprinted with permission from [69].

flow, to promote desolvation of the analyte, and to increase the distance between the nanospray and the MS inlet (Figure 5).

The developed device was tested for the analysis of rat kidney tissue sections, allowing us to obtain an improvement in sensitivity of about 1-3 orders of magnitude compared to the conventional setup. In addition, ion images characterized by high contrast, suitable for more intricate studies of metabolite distribution in biological samples, were obtained. A more complete desolvation of the analytes and reduced ionization suppression were additional features of the proposed device.

\subsection{Extractive ElectrosprayIonization-Mass Spectrometry and Laser} Ablation Electrospray Ionization-Mass Spectrometry. Extractive electrospray ionization (EESI) has been introduced in 2006 by Chen et al. [79]. It is based on the use of two different sprayers: the ESI sprayer generates a charged solvent spray, whereas the sample sprayer has the function to nebulize the sample solution from an infusion pump. The analytes are ionized in the collision area of the two sprays, and then, they are collected by the MS inlet.

The ionization mechanism of the EESI ion source was studied by Wang et al. [80], and different MS-based methods for the analysis of organic aerosols [81], drugs [82], pesticides [83], amino acids [84], and biomarkers [85] in different matrices were developed in the recent years.

LAESI-MS is another ambient ionization technique developed in 2007 by Nemes and Vertes [12]. Since most cells used for biomedical applications are cultured adherently, the use of LAESI-MS was proposed to analyze adherent cells directly onto the culture surface, thus avoiding chemical modification deriving from their detachment [86]. In order to reduce the LAESI spot size, the authors applied a transmission geometry- (tg-) LAESI and incorporated an objective with a high numerical aperture, thus achieving spot sizes in the $10-20 \mu \mathrm{m}$ range. This technique (Figure 6) was tested for the analysis of adherent versus suspended mammal cells, highlighting a difference in the metabolite compositions, thus proving that the cell detachment usually performed is able to produce chemical changes. On the contrary, tg-LAESI-MS allowed us to analyze directly the cells in their native state and, due to the smaller spot size, to reduce the sampled cell population by a factor of 20 .

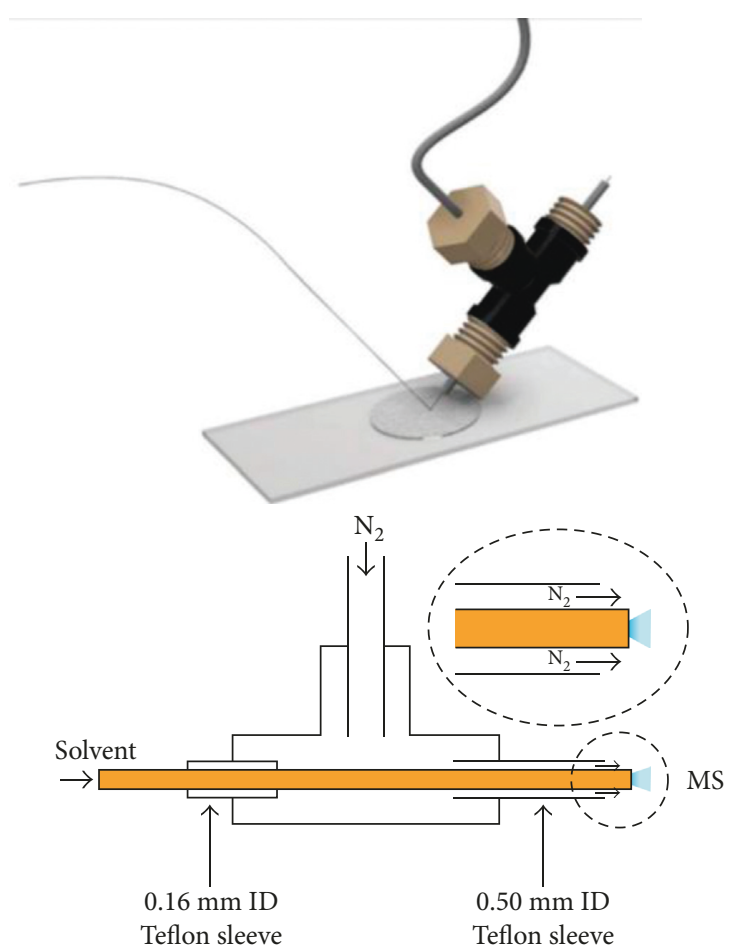

Figure 5: Picture and schematic representation of the pneumatically assisted nano-DESI ionization source. Reprinted with permission from [78].

Optical microscopy combined with LAESI-MS has been suggested by Compton et al. [87] in order to acquire both morphological and chemical information from tissue sampling. In the developed instrumental setup, laser ablation occurred inside a chamber placed under an optical microscope: the ablated particulates generated by the laser were transported through a transfer tube by using nitrogen as carrier gas and finally ionized by the ESI spray.

In order to compare the performances of the developed prototype with those of the conventional LAESI-MS, plant tissues were analyzed. In comparison with conventional LAESI, the developed technique was characterized by reduced sensitivity and dynamic range; however, these features were still sufficient for the analysis and characterization of numerous metabolites and lipids in different spatial regions of biological tissues. 


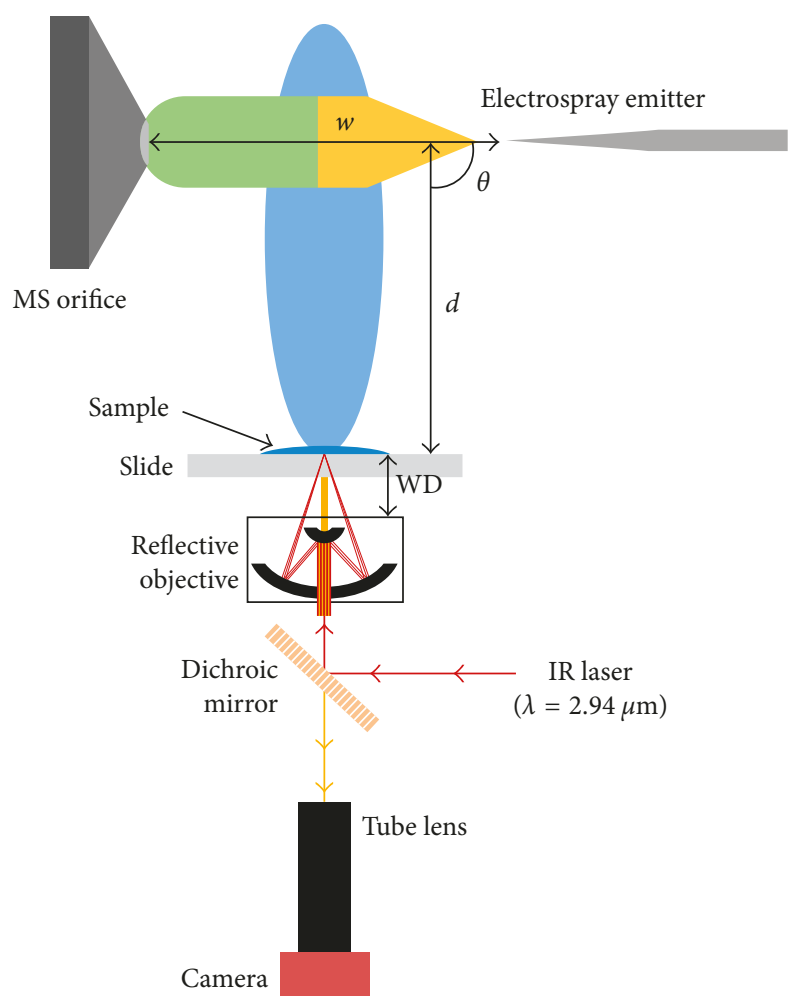

FIgURE 6: Schematic representation of tg-LAESI-MS. Reprinted with permission from [86].

3.3. Paper Spray Ionization-Mass Spectrometry. In the last ten years, approaches based on the direct ionization from solid substrates, such as paper spray, probe-based spray, leaf spray, and tissue spray, strongly increased. All these techniques are characterized by the generation of an electrospray directly from a probe. The analytes in the samples can either be ionized directly upon the substrate surface or be extracted on a probe and subsequently ionized within few minutes, thus boosting analysis speed.

Innovations in PSI-MS have been described by Duarte et al. [88] and Salentijin et al. [89] who developed 3D-printed cartridges in order to obtain a solvent reservoir, thus allowing us to prolong the spray generation from the paper tip. A supporting prototype able to automatically perform PSI-MS analysis of a great number of samples, suitable for high-throughput applications, has been designed by Shen et al. [90]. Finally, the coupling of SFME with PSI-MS for the rapid analysis of macrolide antibiotics at the trace level in biological samples such as whole blood, milk, and other body fluids has been proposed in a study carried out by Deng et al. [91]. The same approach was applied for the detection of perfluorooctanesulfonic acid and perfluorooctanoic acid from Daphnia magna body fluids. After the SFME extraction, the organic extract was simply spotted on the PSI paper.

Excellent results were achieved in terms of linearity range (5-500 and $0.5-50 \mathrm{ng} / \mathrm{ml}$ range for antibiotics and perfluorocompounds, resp.) and sensitivity (LOQs $0.3-1.3$ and $0.03-0.30 \mathrm{ng} / \mathrm{ml}$ for antibiotics and perfluorocompounds, resp.). Recovery rates always higher than $85 \%$ were obtained.
A novel paper spray cartridge with an integrated solidphase extraction column has been developed by Zhang and Manicke [92]. The system was designed in order to perform on the same device the extraction, preconcentration, and ionization of the analytes from complex matrices such as blood or plasma. The cartridge was divided into two parts: the bottom one containing the absorbent waste pad and the paper spray substrate and the top one presenting a hole to host the solid-phase extraction (SPE) column. The procedure for performing paper spray analysis is the following: (i) the samples are loaded onto the SPE column (sample volume from $10 \mu \mathrm{l}$ up to hundreds of microliters); (ii) the unbounded compounds pass through the SPE column and are absorbed by the waste paper pad; (iii) after sliding the top part of the cartridge to the paper spray substrate, the analytes retained on the SPE column are eluted and analyzed by PSIMS. The analytical performances in terms of detection and quantitation limits, recovery, and ionization suppression were evaluated for carbamazepine, atenolol, sulfamethazine, diazepam, and alprazolam. The SPE cartridge allowed both the selective enrichment of the targeted analytes from large sample volumes (up to hundreds of $\mu \mathrm{l}$ ) and the removal of interfering compounds, thus enhancing signal intensities. Compared to direct PSI, the proposed method allowed us to improve quantitation limits by a factor of 14-70, obtaining limits in the $0.2-7 \mathrm{ng} / \mathrm{ml}$ range.

3.4. Wooden-Tip Electrospray Ionization-Mass Spectrometry. Wooden-tip electrospray ionization-mass spectrometry (WTESI-MS) is a rapid, in situ, and direct ambient technique based on the use of a wooden tip as a sampling and ionization needle. The tip is dipped into the liquid solution/matrix, and after extraction, it is directly used as an ESI probe by applying a high voltage and spray solvent. The analytes enriched on the tip are desorbed and ionized under ambient and open-air conditions.

This method has been successfully applied by Yang et al. [93] for the analysis of pesticides, toxicants, date rape drugs, and illicit additives in various food samples. The capabilities of untargeted WT-ESI-MS analysis for the identification of the sources of plant materials by using a multivariate statistic approach were exploited by Xin et al. [94], whereas Yang and Deng [95] used an internal standard WT-ESI-MS-based method to obtain the fingerprint mass spectra for rapid quality assessment and control of Shuang-Huang-Lian oral fluid, an herbal preparation registered by Chinese Pharmacopoeia. By using the internal standard and principal component analysis (PCA), it was possible to obtain the fingerprints of samples from different manufacturers. A bamboo pen nib shaped and used for sample loading and an ESI probe for the determination of 4-chloro-amphetamine was developed by Chen et al. [96], resulting in lower detection limits compared to PSI-MS and traditional WT-ESIMS analyses.

Similarly, a WT-ESI-MS method combined with different nonpolar solvents for the detection of native proteins and protein complexes directly from raw biological samples has been proposed by $\mathrm{Hu}$ and Yao [97]. 
The applicability of field-induced wooden-tip electrospray ionization-mass spectrometry (FI-WT-ESI-MS) for high-throughput analysis of herbal medicines has been proved by Yang et al. [98]. Field-induced ESI was performed by applying a high voltage on the MS inlet, thus allowing the creation of a strong electric field between the capillary emitter and MS inlet to induce ESI from the sample solution. A high-throughput analysis device was developed by the application of sample-contactless high voltage on the MS inlet. In addition, the switch between positive and negative ion detection modes can be readily accomplished, thus providing complete MS information of the analyzed samples. This approach allowed us to boost the analysis speed: $6 \mathrm{~s}$ per sample was sufficient to perform the analyses.

The proposed method was applied for the rapid determination of various active ingredients in different raw herbs and herbal medicines. The obtained mass spectra were used as fingerprints for tracing the origins, establishing the authenticity and assessing the quality of herbal medicines.

Very recently, a novel and noninvasive sampling method using a watercolor pen (brush) rinsed with ethanol as a sampling tool to collect analytes from the eyelids of volunteers has been evaluated [99]. The brush was placed between the mass inlet and the ESI plume, thus allowing the desorption and ionization of the analytes. The results achieved proved the suitability of the developed technique for the semiquantitative determination of caffeine and its metabolites in eyelid samples.

3.5. Miscellaneous. Following the method developed by Pan et al. [100], based on the use of a single probe inserted into a single cell for sampling intracellular compounds by realtime MS analysis, Chen et al. [52] described a novel method for single cell analysis and lipid profiling by combining dropon-demand inkjet cell printing and probe electrospray ionization-mass spectrometry (PESI-MS). Droplets containing single cells were generated from a cell suspension by inkjet sampling, precisely dripped onto the tungsten tip of the ESI needle, and sprayed under a high-voltage electric field. Cellular lipid fingerprints were then obtained by MS detection. The analytes from eight types of cells were detected, and PCA analysis was performed in order to differentiate the samples. The proposed platform was demonstrated to be suitable for the direct MS profiling of single-cell lipid species without derivatization or the labeling procedure.

A method for the direct characterization of metals in solid samples using electrospray laser desorption ionizationmass spectrometry (ELDI-MS) has been developed by Shiea et al. [101]. The main advantages over classic approaches were related both to the absence of sample pretreatment and to the presence of very short analysis time. Mixtures of metal-EDTA complexes were applied on a stainless steel plate and submitted to ELDI-MS analysis. The capabilities of the technique were initially tested by spotting the metalEDTA complexes on a solid probe and performing laser ablation of the material. The ablated analytes, present as EDTA complexes, were ionized in the electrospray plume.
Further experiments were carried out by deposing the sample on the probe surface while maintaining the EDTA in the ESI spray solvent (functional electrospray). Excellent results in terms of sensitivity were achieved, thus proving method reliability for the rapid analysis of metal substrates without sample preparation.

An electrostatic spray ionization (ESTASI) method for the analysis of samples deposited in or onto an insulating substrate has been proposed by Qiao and coworkers [102]. In this study, the ionization of the analytes is induced by a capacitive contactless coupling between the electrode and the sample: by applying a pulse high voltage to the electrode, an electrostatic charging of the sample occurs, leading to a bipolar spray pulse. When the applied voltage is positive, the bipolar spray pulse consists first of cations, followed by anion production. The instrumental setup can be modified in order to obtain ion emission from samples in a silica capillary, in a disposable pipette tip, and in a polymer microchannel or deposited as droplets onto an insulating poly(methyl methacrylate) plate presenting wells or hydrophilic patches. This technique proved to be suitable for the analysis of peptides and proteins.

\section{Materials for Spray-Based Ionization Techniques}

The development of new materials is a field of increasing interest in order to enhance the performances of analytical methods. Both high selectivity and increased ionization efficiency are demanded to detect analytes at trace levels in complex matrices and to shorten analysis time. Different studies have been published dealing with the development of novel surfaces for ambient MS. A brief overview on the most recent materials developed for spray-based ionization techniques with particular attention to DESI-MS and PSIMS applications is described in the next paragraphs.

4.1. Materials for DESI-MS. In 2005, Takats et al. [103] demonstrated that the DESI ion source is strongly influenced by the dielectric constants of both the substrate material and the spray.

The effect of surface chemistry in the DESI ionization mechanism has been deeply investigated by Penna and coworkers [104]: the performances of different glass substrates obtained by the sol-gel technology and functionalized by using different alkylsilanes were tested and compared in terms of ionization efficiency. The substrates were characterized in terms of surface free energy and wettability. Owing to their different polarity, melamine, tetracycline, and lincomycin were used as model compounds. A significant decrease in the ionization efficiency was observed when more hydrophilic surfaces were used, thus demonstrating the pivotal role of both hydrophobicity and wettability to increase the performances of DESI-MS experiments.

More recently, a 3D printed polylactic (PLA) supports in order to detect insulin and gentamicin in chitosan gels have been proposed by Elviri and coauthors [105]. By using 3D printing, hemispherical wells were created, thus allowing us 


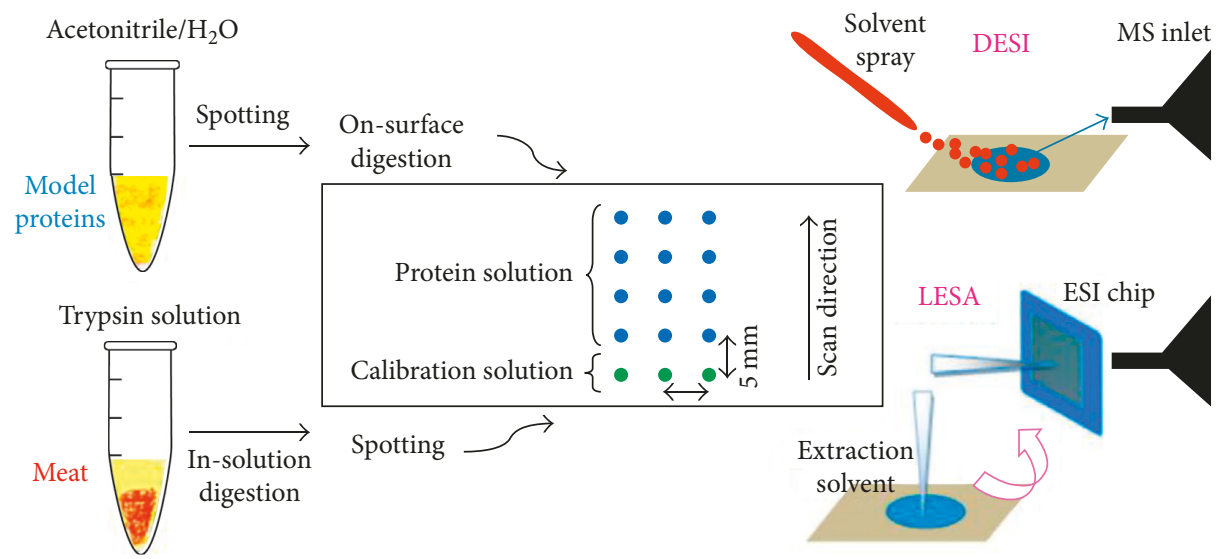

FIGURE 7: Schematic representation of the procedure proposed by Montowska et al. Reprinted with permission from [106].

to obtain DESI-MS responses five times higher than those achieved by using PTFE commercial slides. Improvement in terms of signal stability was also achieved, thus suggesting the capability of $3 \mathrm{D}$ printing technology to improve the desorption step in DESI-MS.

Novel substrates have been proposed also for proteomics and peptidomics: the capability of Permanox slides for both DESI-MS and liquid extraction surface analysis-mass spectrometry (LESA-MS) of skeletal muscle proteins obtained from a mixture of standard proteins and raw meat has been discussed in a recent study [106]. The proposed method is schematically reported in Figure 7.

In both cases, good responses were obtained with LESA-MS characterized by higher sensitivity and stability with respect to the DESI-MS approach. Finally, multivariate data analysis allowed the correct discrimination among different meat classes.

Rapid and simple analyses represent a key parameter in proteomics: an interesting study was carried out by Dulay et al. in 2015 [107]. Two hybrid organic-inorganic organosiloxane polymers functionalized by immobilized trypsin (T-OSX) for the on-surface and in situ digestion of four model proteins, that is, melittin, cytochrome c, myoglobin, and bovine serum albumin, were developed and tested under DESI-MS and nano-DESI-MS conditions. The silica polymers were obtained via sol-gel technique using methyltrimethoxysilane and dimethyl-dimethoxysilane as precursors. The OSX polymers were derivatized with trimethoxysilylbutyraldehyde and functionalized with different amounts of trypsin. In both cases, despite the low enzyme-to-substrate ratios, the achieved results proved the suitability of the developed substrates to allow on-surface protein digestion followed by direct DESI-MS analyses obtaining sequence coverages in the $65-100 \%$ range.

Taking into account that DESI-MS analyses can be performed also on liquid samples, an improvement of the apparatus commonly used for this kind of analyses has been proposed [108] by replacing the sample transfer silica capillary with a trap column filled with chromatographic stationary phase materials, such as $\mathrm{C}_{4}$ and $\mathrm{C}_{18}$. The proposed system proved to be suitable for trace analyses of both organic compounds and biomolecules such as proteins/peptides in complex matrices in the presence of high salt content. Another interesting feature was related to the covalent functionalization of the inner wall of the sample transfer capillary with enzymes, thus allowing the fast and online digestion of proteins.

Noticeable applications of DESI-MS are based on its coupling with new sampling devices and extraction and separation techniques to develop methods for highthroughput analyses.

The microfabrication of ultrathin-layer chromatography (UTLC) plates via conformal low-pressure chemical vapor deposition of silicon nitride onto patterned carbon nanotube (CNT) scaffolds, acting as surface templates, has been described by Kanyal et al. [109]. The plates were heated and oxidized to both remove the CNTs and convert $\mathrm{Si}_{3} \mathrm{~N}_{4}$ into silica; finally, the plates were hydroxylated in aqueous ammonium hydroxide. The resulting UTLC phases did not show any distortion of the microfeatures and were characterized by a higher robustness in comparison to high-pressure TLC plates. Good results in terms of chromatographic performances were observed obtaining a faster separation when mixtures of lipophilic, water-soluble, and fluorescent dyes are to be analyzed. A strong reduction in terms of mobile-phase consumption and an enhanced lifetime were observed. Finally, the UPTLC plates were submitted to both DESI-MSI and direct analysis in real-time (DART)-MS analyses, showing a good compatibility with common ambient desorption and ionization techniques.

In the same year, Ewing et al. [110] described the DESIMS detection of the low vapor pressure nerve agent simulant triethyl phosphate. The analyte was previously adsorbed onto silica gel, forming a very fine particulate that was collected by using a sticky screen sampler. The device was characterized by a stainless steel screen presenting a partially polymerized polydimethylsiloxane (PDMS) coating. The quantitative collection of the particulate sample from a contaminated surface was achieved by interfacing the sticky screen sampler with a bioaerosol collector. Finally, the sticky screen was placed onto a moveable platform mounted in front of the DESI-MS instrument, thus allowing a reproducible sample introduction system able to minimize sampling errors. DESI-MS analyses performed directly on the PDMS coating allowed us to obtain a very low detection limit suitable for trace detection. 
Electrospun nylon-6 nanofiber mats for DESI-MS analysis and imprint imaging have been developed by Hemalatha et al. [111]. The nanofibers were developed by needleless electrospinning: nylon-6 was dissolved in formic acid and the solution was electrospun at room temperature. Uniform mats of varying thicknesses composed of $\sim 200 \mathrm{~nm}$ diameter fibers were obtained: the properties of these materials can be tuned by varying spinning conditions and surface functionalization. As model compounds, dyes and the extract of periwinkle flower were spotted onto the nylon nanofiber mat, thus obtaining a uniform coating of the fibers. DESI-MS analysis allowed us to obtain spectra without polymer interference and reproducible DESI-MS images. The authors also demonstrated that compounds of interest could be incorporated into nanofibers during their formation by using as model compounds the crude methanol extract of periwinkle flower and tetraphenylphosphonium bromide (TPPB). The major metabolites of periwinkle were identified by DESI-MS, even though the spectrum was different compared to that obtained by spotting the sample. TPPB was detected with no nylon interference. The authors demonstrated the possibility of imprinting patterns made of printing inks, plant parts, and fungal growth on fruits on the nanofiber mats. Metabolites were identified by DESI-MS. The results highlighted that electrospun nanofiber mats could be considered as smart surfaces to capture diverse classes of compounds for rapid detection or to imprint imaging under ambient conditions.

4.2. Materials for PSI-MS. Although traditional paper spray ionization is performed on the filtering and chromatographic paper [11,112-115], researchers have focused their attention to the development of new functionalized substrates in order to obtain substrates characterized by enhanced selectivity and sensitivity.

Very recently, Lai et al. [116] compared the ionization performances of different paper-like substrates obtained from both natural fibers and synthetic fibers, namely, gampi paper, Tengujou paper (natural), polycarbonate, polylactic acid, and poly-l-lactic acid (PLLA) (synthetic), with those of traditional chromatographic paper for the analysis of designer drugs. The surface characterization of the developed materials showed the presence of different surface morphologies able to affect PSI capabilities: gampi paper and PLLA nanofibers, characterized by a tough and extremely thin structure, were able to promote signal enhancement, thus allowing us to reach lower limits of detection. These findings could be explained by taking into account the reduced thickness of the used papers: by operating under the described conditions, a rapid evaporation of the sample molecules occurs, thus increasing the speed of the ionization process.

The analytical performances of paper with paraffin barrier (PS-PB) for the PSI-MS detection of hydroxymethylfurfural (HMF) and sugars like glucose and xylose in sugarcane liquors have been tested by Colletes et al. [117]. Microfluidic hydrophobic channels were obtained using paraffin barriers on paper substrates, thus delimiting a region for inducing the sample inlet into the mass spectrometer. The proposed stamping method allowed rapid prototyping of microfluidic paper-based analytical devices, without the need of sophisticated instrumentation. Different types of papers were investigated: an increase of the PSI-MS responses of xylose and glucose as a function of the decrease of porosity of the paper substrate was observed. PS-PB showed the best performance compared to the conventional paper and paper with two rounded corners. PS-PB was applied to detect sugars and their inhibitors in liquors from a second-generation ethanol process, thus obtaining excellent results in terms of linearity (over two orders of magnitude) and limits of detection and quantification.

Another interesting study carried out by Zhang et al. [118] proposed the use of commercially available silicacoated paper in order to increase the PSI-MS responses of therapeutic drugs in dried blood spots. The presence of silica gel particles in the cellulosic framework of the silica-coated paper produced a reduced diffusion of the blood through the substrate, thus leading to a higher percentage of blood sample available on the top side of the substrate. By operating under the optimized conditions, that is, by using dichloromethane/isopropanol $(9: 1 \mathrm{v} / \mathrm{v})$ as a spray solvent mixture, limits of quantitation of about $0.1 \mathrm{ng} / \mathrm{mL}$ were achieved, with a sensitivity gain of 5-50-fold in comparison to chromatography papers.

CNT-coated filter paper for low-potential PSI-MS analysis of different organic molecules has been used by Narayanan and coauthors [119]: by applying a voltage of $3 \mathrm{~V}$, full-range mass spectra similar to those obtained by conventional ESI at $3 \mathrm{kV}$ could be recorded. The advantage of the proposed material relies on the use of very mild conditions for the ionization of the analytes, thus allowing the detection of compounds that could be easily oxidized. The performances of the proposed analytical method were assessed for a wide range of volatile and nonvolatile compounds, such as amino acids, antibiotics, and pesticides in different matrices.

Very recently, Wei et al. [120] synthesized graphene oxide (GO) nanosheet-modified $\mathrm{N}^{+}$-nylon membrane (GOM) for the extraction of malachite green (MG), a highly toxic disinfectant, and its metabolites in liquid samples and fish meat. GO nanosheets are characterized by a very high surface area $\left(\sim 800 \mathrm{~m}^{2} / \mathrm{g}\right)$, suitable for MG adsorption via $\pi-\pi$ stacking and electrostatic interactions. GOM was obtained by selfdeposition of $\mathrm{GO}$ thin films onto $\mathrm{N}^{+}$-nylon membranes. The material was tested both as a direct spray ionization substrate and as an LDI-MS probe. The latter application resulted in no significant response, whereas the coupling between GOM and direct spray ionization allowed the quantitation of $\mathrm{MG}$ and its metabolites at nanomolar levels with extraction recoveries higher than $98 \%$.

An improvement of the performances of PSI-MS in terms of sensitivity has also been achieved in a study dealing with the use of a paper substrate functionalized with urea [121]. Triangles of chromatography paper were treated with 1-[3-(trimethoxysilyl)propyl]urea to create an anion capture layer. The authors demonstrated that the urea-modified paper is an excellent substrate for PSI-MS since it is able 
to reduce ionization suppression caused by anions and highly polar compounds in the negative-ion MS mode. These findings are of pivotal importance for the analysis of biological samples like urine, blood, and plant extracts.

A selective substrate for PSI-MS, based on the use of molecularly imprinted polymers (MIPs), has been proposed by Pereira et al. [122]. More precisely, a membrane spray ionization method, combining MIP extraction and PSI-MS analysis, was developed and tested for the determination of diuron and 2,4-dichlorophenoxyacetic acid from apple, banana, and grape methanolic extracts. Being used as PSI substrates, MIPs were synthesized directly on a cellulose membrane: the bulk of the MIP was made by ethylene glycol dimethacrylate, using monuron and 2,4,5-trichlorophenoxyacetic acid as templating agents. After extraction, the MIP membranes were washed to remove matrix interferences and tested as PSI-MS substrates using methanol as a spray solvent. The use of these novel materials allowed us to obtain signal intensities of the targeted analytes far higher than those obtained by nonimprinted polymers with detection limits in the $\mu \mathrm{g} / \mathrm{l}$ range.

Bills and Manicke [123] developed a disposable paper spray cartridge containing a plasma fractionation membrane to perform on-cartridge plasma fractionation from whole blood samples. Three commercially available blood fractionation membranes, made of different materials, ranging from polymers to natural and synthetic fibers, that is, Vivid polysulfone membrane, NoviPlex plasma fractionation card, and CytoSep, were tested. Even though all the materials were capable of interacting with plasma samples with low levels of cell lysis, difficulties in terms of drug extraction were observed. In particular, Vivid polysulfone membrane and NoviPlex plasma fractionation card exhibited a high binding capability (over 30\%) for all the tested drugs, whereas CytoSep showed a lower binding affinity $(<17 \%)$ only for two out of five drugs. A drawback of the developed device was also related to the poor fractionation efficiency, as measured by the red blood cell content in the fractionated plasma. Quantitative analysis of plasma using PSI-MS provided results closed to those obtained by HPLC-MS without the need of offline extraction or chromatography separation.

A new zero volt-paper spray ionization (ZV-PSI) has been developed recently by Wleklinski et al. [124]: this approach is based on the generation of the electrospray by the action of the pneumatic force of the vacuum at the MS inlet. ZV-PSI analyses were performed over a large variety of samples, including tributylamine, cocaine, terabutylammonium iodide, 3,5-dinitrobenzoic acid, fludioxonil, and sodium tetraphenylborate. In comparison to classic PSI-MS, the achieved results showed a strong decrease of signal intensities for all the investigated analytes. Although the range of analytes useful for ZV-PSI-MS analysis resulted to be very similar to standard PSI-MS, differences in mass spectra were obtained. The observed behavior was related to the ionization mechanism of the proposed approach, which is strongly related to the effects of analyte surface activity. By using a Monte Carlo simulation, the mechanism regarding the formation of ions from initially uncharged droplets was also explained, thus allowing us to predict detection limits very closed to those observed experimentally and to calculate the relative surface activity of both positive and negative ions.

\subsection{Materials for Wooden-Tip Electrospray Ionization-Mass} Spectrometry. Surface-coated wooden-tip electrospray ionization-mass spectrometry (SCWT-ESI-MS) is a new technique based on the use of a functionalized wooden needle, acting both as extraction/enrichment phase and ESI probe. By using this strategy, the tip is coated by a proper sorbent for highly selective enrichment of targeted compounds from complex matrices, thus making it suitable for analyses at ultratrace levels. Luan suggested the use of a SCWT-ESI-MS technique to detect different analytes in complex matrices $[125,126]$.

The SCWT-ESI-MS technique has been applied for the detection of perfluorinated compounds (PFCs) in complex environmental and biological samples at ultratrace level [125]. Sharp wooden tips were functionalized via the silanization process by using octadecyltrimethoxysilane and n-octadecyldimethyl[3-(trimethoxysilyl)propyl]ammonium chloride (OTPAC), in order to obtain two different extractive phases, characterized by long alkyl chain. The two phases were tested for the extraction of PFCs spiked water. The OTPAC coating was characterized by the best enrichment capabilities: the extraction is performed not only by the reversed phase, but also by exploiting the ion exchanged adsorption mechanism. Morphological studies of the tip showed a high probe porosity, thus increasing the surface area of the material, and presence of microchannels for transport of the solvent to achieve ambient ionization MS analysis. After method optimization, the probe was tested for the detection of eight different PCFs both in pure water and in complex matrices, that is, lake water, river water, whole blood, and milk. The achieved results proved that the SCWT probe is characterized by outstanding enrichment capabilities, thus being able to enhance method sensitivity by approximately 4000-8000folds and 100-500-folds in aqueous samples and in whole blood and milk samples, respectively. Method validation resulted in good linearity (two orders of magnitude), excellent quantitation limits (in the $0.21-1.98 \mathrm{ng} / \mathrm{l}$ range), and accuracy (recovery rates in the $89-112 \%$ range).

Similarly, a SCWT-ESI-MS-based method has been tested for the rapid and sensitive analysis of trace fluoroquinolone and macrolide antibiotics in water [126]. The wooden probe was functionalized via silanization and sulfonation reactions in order to obtain a sulfo- $\mathrm{C}_{8}$-chain coating able to interact with the analytes with both reversed phase and ion exchange mechanisms. The SCWT-ESI-MS method was then optimized and tested for the extraction of four fluoroquinolone and two macrolide antibiotics in water at trace levels. Method sensitivity allowed us to obtain detection and quantitation limits in the 1.8-4.5 and 5.9$15.1 \mathrm{ng} / \mathrm{l}$ range, respectively. Again, linearity was verified over two orders of magnitude: good precision (RSD <14.3\%) and recovery rates in the $93.6-112.6 \%$ range were other features of the developed method. Finally, the developed method was applied for the analysis of the targeted antibiotics in tap and river water samples. 


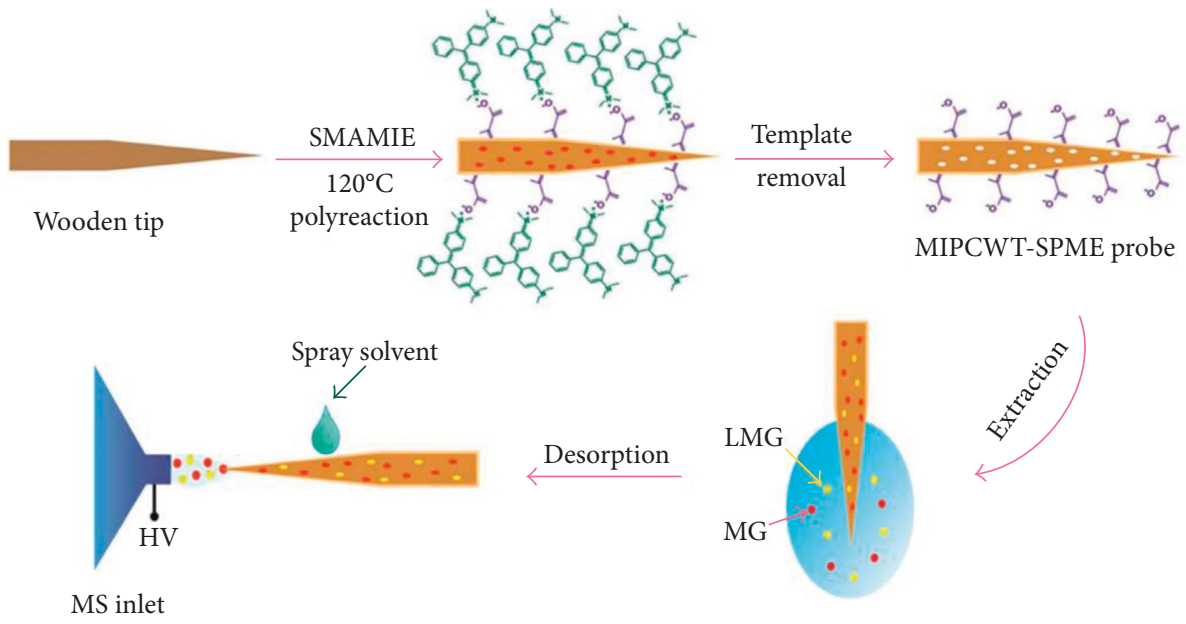

FiguRE 8: Schematic representation of the instrumental setup proposed by Huang. Reprinted with permission from [127].

An interesting approach based on the use of molecularly imprinted polymers as coating for SCWT-ESI-MS has been discussed in a recent study [127]. The coating was synthesized by applying a silicone-modified acrylate molecularly imprinted emulsion onto the tip surface (Figure 8). The developed material was tested for the detection at trace levels of malachite green and its metabolite leucomalachite green in aqueous samples. The MIP-SCWT probe exhibited high enrichment capabilities, allowing us to obtain detection limits at low $\mu \mathrm{g} / \mathrm{l}$ levels. In addition, a good linearity was obtained for both the compounds (three orders of magnitude). The method proved to be suitable for high-throughput analysis and was successfully applied for the analysis of tap water, river water, and fish samples.

4.4. Miscellaneous. Similar to PSI, aluminum-foil mass spectrometry (Al-ESI-MS) was recently developed by $\mathrm{Hu}$ et al. [128]. This technique is based on the use of a household aluminum foil to obtain the spray ionization of the analytes. The $\mathrm{Al}$ foil was cut into triangles, which were folded symmetrically to obtain a mini-reservoir for the sample solution, and was connected to the high voltage supply of the mass spectrometer. The proposed technique was tested for the direct analysis of a wide variety of complex matrices, namely, energetic beverages, urine, skincare and medical creams, and herbal medicines. The inert, hydrophobic and impermeable surface of the $\mathrm{Al}$ foil allowed effective on-target extraction of solid samples and on-target sample clean-up, that is, removal of salts, adulterants, and detergents from proteins and peptides. Being $\mathrm{Al}$ an excellent heat conductor, the direct monitoring of thermal reactions, such as thermal denaturation of proteins, can be performed in an easy way by Al-ESI-MS.

In a different research study, ESTASI was applied to identify and quantify different compounds from silica gel surfaces, via direct coupling with TLC [129]. The sample spots separated by TLC were analyzed by ESTASI-MSI. The analyses were performed on both drug molecules, using normal phase TLC, and dyes using reversed phase C18 TLC plates, thus guaranteeing the analyses of compounds characterized by very different polarity. After sample separation, the hydrophobic substrate was coated with chlorotrimethylsilane to form hydrophobic surfaces, suitable for ESTASI analysis. Both TLC plates were considered ideal substrates for in situ characterization of samples by using ESTASI-MS, with efficient analyte extraction and separation. In addition, the capability of removing interfering compounds such as salts increased method performance, thus allowing the detection of the investigated analytes at trace levels.

\section{MS-Based Approaches for Food Analysis}

The demand for safe and high-quality foods has significantly increased in recent years. Food safety and quality have become of greater importance, and the governments of many countries have increased the amount of relevant legislation and demands for food authentication [130]. In consequence, the development of more robust, efficient, cost-effective, and powerful analytical methodologies is continuously needed in order to face these requirements. MS is one of the most suitable techniques because it is featured by excellent specificity, sensitivity, and throughput [131]. MS has been widely used in food safety and quality analysis, and recent advances in MS can provide faster and more accurate methods able to offer better qualitative and quantitative results. Additionally, coupling mass analyzers with separation techniques, such as liquid chromatography (LC-MS) and gas chromatography (GC-MS), have significantly improved food analysis for screening, identification, structural characterization, and quantitation purposes.

One of the most challenges in the application of MS in food analysis, especially in detection of contaminants, is sample preparation because foods are considered very complex matrices in which some natural components can negatively influence the analysis of the targeted compounds. Traditional methods for sample extraction include solidliquid extraction (SLE), liquid-liquid extraction (LLE), and solid-phase extraction (SPE). More recent is the use of solidphase microextraction (SPME), pressurized liquid extraction 
(PLE), and QuEChERS (quick easy cheap effective rugged safe) [132]. The introduction of high-resolution mass spectrometers, which provide extremely high selectivity and sensitivity, or other emerging MS strategies such as ambientionization MS, direct food analysis, and matrix-assisted lased desorption ionization-time of flight-mass spectrometry (MALDI-TOF-MS) profiling and imaging, has strongly reduced sample preprocessing.

According to the PubMed database, since 2012 about 20000 publications dealing with developed MS-based methods for food safety and quality purposes are available. In this section, we do not intend to provide an exhaustive revision of all published studies, but an overview of the most important MS techniques proposed to evaluate food safety and quality.

5.1. MS-Based Approaches for Food-Safety Assessment. The main purpose of food analysis is to ensure food safety, thus requiring the development of accurate and reliable methodologies for the detection of microbial-related spoilage, determination of allergens, detection of environmental contaminants as well as banned external compounds, or the assessment of the occurrence of natural toxins. These methods are strongly influenced by current legislation, which establishes the requirements that an analytical method must meet for an unequivocal identification and quantification of a controlled substance in food samples, as well as the maximum residue limits (MRLs) on certain substances [133].

Being able to allow the quantification of known compounds with great selectivity and sensitivity, tandem MS detection is one of the most frequently utilized analytical approaches to determine contaminants in foods. Triple quadrupole $(\mathrm{QqQ})$ mass spectrometers, running under multiple reaction monitoring (MRM) mode, are the most popular instruments for detecting contaminants in food. This detection procedure allows us to verify the compliance with European legislation on banned and controlled substances in foods [133].

Since 2010, numerous researches have used this methodology to detect pesticides in several fruits and vegetables, such as tomato [134-137], orange [138], mandarin [139], rice and red pepper [139-141], avocado [142, 143], apples and cucumbers [144, 145], mango [146], tea [147], lettuce [148], grains and cereals [149-152], soybean products [153], groundnut oil [154], and wines [155]. The same methodology was also used in the identification and quantification of veterinary drug residues in shellfish [156], meat $[157,158]$, eggs and milk [158], and contaminants from food contact materials [159].

Triple quadrupoles in MRM mode is also the mostextended approach to detect and quantify toxins and pathogens in food. These pathogens can contaminate foods directly or indirectly, through the productions of toxins. The control of toxin and pathogen levels is extremely important, since the consequences on health due to their contamination of food may be very serious. Following this approach, food products such as nuts [160], maize [161], shellfish [162], tomato [163], beer [164], and multicereal baby food [165] were analyzed.
Almost all these applications combine QqQ-MS with LC or GC separation methods. In some cases, LC- and GCbased techniques were also coupled with other types of MS analyzers such as ion traps (ITs) or quadrupole-linear ion traps (Q-LITs), TOF, or Q-TOF to determine food contaminants [166-168].

Multidimensional procedures allowed us to increase resolving power and separation capabilities that can be beneficial for subsequent MS-based detection, considering that the targeted compounds can reach the detector more separated in time. This is the case of comprehensive two-dimensional gas chromatography $(\mathrm{GC} \times \mathrm{GC})$ that has been coupled to a TOFMS analyzer to determine dioxin-related pollutants in complex food samples [169], to screen 68 pesticide residues in oilseed [170], or to detect and to quantify different polychlorobiphenyls (PCBs), polybrominated diphenyl ethers (PBDEs), and PAHs in fish samples [171].

More recently, HRMS analyzers, typically Q-TOF, Orbitrap-MS, and Fourier-transform ion cyclotron resonance- (FT-ICR-) MS, have been used in the field of food safety. These instruments are characterized by high resolution (100,000-1,000,000 FWHM) and high mass precision (1-2 ppm, allowing discrimination between isobaric interferences and ions of interest), thus making possible the screening of unknown compounds with a full MS scan and the construction of databases for targeted compounds. For instance, UPLC-Orbitrap-MS was used to create a database of more than 350 compounds in honey [172]. These databases included different classes of pesticides and veterinary drugs and allowed simultaneous screening of analytes and identification and quantitation of detected compounds in different honey samples. Similar UPLC-HRMS approaches have been lately used to create an accurate-mass database including the fragmentation of more than 600 different food contaminants, such as pesticides, veterinary drug residues, mycotoxins, and perfluoroalkyl substances [173]. Since the particle size of the stationary phase in UPLC is significantly lower than that observed in HPLC, UPLC yields higher speed, better resolution, increased sensitivity, and better peak capacity. Additional examples are related to the development of LC-Orbitrap-MS-based methods for pesticide screening in vegetables and fruits [174], as well as for the analysis of 18 selected mycotoxins in baby food [175].

Ambient MS-based techniques have also been widely applied for food safety purposes: different ionization techniques have been used like LAESI-MS to detect neurotoxin domoic acid in shellfish [176], DESI-MS for the rapid detection of shellfish poisoning toxins in mussels [39], and PSI-MS for the determination of pesticides in fruits and vegetables [114].

5.2. MS-Based Approaches for Food Quality Assessment. Besides food safety, food quality is one of the main concerns of the modern food industry. Food quality encompasses multiple factors, since food authentication and adulteration of food characteristics include food ingredients, such as lipids, proteins, oligosaccharides, vitamins, and carbohydrates, and additives, such as preservatives, antioxidants, and chemicals used for flavor, color, and odor. As a consequence, the 
evaluation of food quality usually represents a very complex task that needs to consider multiple aspects to achieve the appropriate food quality. Food composition, aroma, flavor, or nutritional properties are among the most important features that need to be evaluated in food quality assessments.

Several MS-based approaches have been used to determine food quality. The most recent publications have mainly used nontargeted MS-based approaches, which very often included the coupling LC-MS and/or GC-MS.

Among LC-MS analytical methods, LC-HRMS techniques have been used for quality evaluation of raw turmeric form different regions [177], for the discrimination of grapes according to plant sterol content [178], for the analysis of the metabolome of the Graciano Vitis vinifera wine variety [179], and for the investigation of the quality and authenticity of saffron [180] and strawberries . Moreover, methods based on the UPLC combined with HRMS were developed to assess the authentication and the evaluation of possible adulterations in saffron [181] and fruits juices [182, 183]. The last two methods rely on the feasibility of the application of the UPLC-QToF platform to perform both nontargeted and targeted methods to select potential biomarkers, which should make it possible to develop a targeted method (less sophisticated instrumentation and simpler data analysis) for routine analysis. Similarly, the combination of nontargeted and targeted methods was reported for the qualitative analysis of curcuminoids in turmeric [184]. In this case, nontargeted analysis was performed by using LC-QTOFMS/MS and the targeted approach by LC-QTRAP-MS/MS.

In the LC-MS-based approaches devoted to food quality assessment, it is noteworthy to highlight the use of hydrophilic interaction chromatography (HILIC), especially in metabolomics approaches. HILIC columns allow profiling highly polar and hydrophilic compounds providing complementary metabolic information to reversed-phase LC. In spite of several caveats associated to HILIC, such as variability in retention times, low peak efficiency, and long re-equilibration times after gradient elution, this methodology has been successfully used to analyze contamination and degradation of infant formulas [185], to separate and detect marine toxins [186], or to identify biomarkers of meat quality [187].

GC-MS-based approaches have also been widely used to evaluate food quality. In these approaches, GC was coupled to a huge diversity of mass analyzers: from simple MS instruments, like quadrupole (Q) [188-192], IT [193], to highresolution instruments [194-198], as well as hybrid analyzers [199-201]. Studies of the effect of volatile compounds for the classification of saffron based on the concentration of biomarkers [188], classification of olive oils on the basis of their quality parameters [200], the establishment of differences between wine grape cultivars [194], or the detection of milk or meat adulteration $[78,190]$ are only some of examples relying on the use of GC-MS platforms in food quality analysis.

Besides the much more common LC-MS and GC-MS platforms to assess food quality, comprehensive twodimensional GC [202] and CE methods [186] coupled to TOF analyzers have also been applied. GC $\times$ GC allowed the creation of a panel of biomarkers of rice flavor quality through establishing associations between volatile metabolites and perception of rice aroma [202]. These results are valuable for breeding programs since they can be used to choose pleasant rice aromas. In the latter, the feasibility of using a polymer-coatedcapillary for the separation of anionic metabolites in both orange juice and wine has been demonstrated [186]. It offers a complementary coverage of the metabolome of these samples to those provided by other analytical techniques.

In addition to spray-based ionization techniques [203-206], mass spectrometry imaging (MSI) is another useful technique for food safety and quality control through monitoring the spatial distribution of bioactive components and contaminants in food samples. Until recently, MSI was largely performed with MALDI. MALDI-TOF-MSI was successfully applied to investigate the distribution of toxic glycoalkaloids in potato tubers [207], to identify the site of capsaicin in Capsicum fruits [208], and to observe both the tissue site of 10 anthocyanin species in blueberries [209] and the posttranslational modified sites in the alpha-melanocyte-stimulatin hormone for carp and goldfish pituitary tissue, as well as their ratio change under different environmental conditions [210]. Although MALDI-MSI can detect compounds in a tissue section without extraction, purification, separation, or labeling, the slow speed of the analysis and the need for matrix deposition in MALDI-MS are critical disadvantages in food imaging applications because they involve analyte diffusion able to affect the original molecular distribution. The development of various ambient ionization techniques revived interest in MSI because of the direct surface sampling in front of a mass spectrometer with submillimeter resolution and no sample preparation. These techniques permit rapid, direct measurement of compounds present on the condensed sample phase and have become potential analytical tools for direct profile-imaging analysis in an atmospheric pressure environment, thus being particularly useful for foodquality control purposes.

Although the application of these techniques in food analysis is not yet fully established, some examples can be found in literature. As an example, ELDI-MS was applied to obtain the molecular profiling and spatial distribution of particular active components in two edible fungi species [211] as well as alpha-solanine and alpha-chaconine in potato [212].

DESI-MSI was used to reveal the spatial distribution of chlorogenic acids and sucrose across the bean endosperm [213], as well as the spatial and temporal distribution of rohitukine and related compounds during various stages of seed development [214]. LAESI allowed macroscopic and microscopic imaging of pesticides, mycotoxins, and plant metabolites in different matrices [215].

\section{Conclusions}

MS-based techniques represent a highly valuable tool for environmental, bioanalytical, food safety, and food-quality control purposes and their application in these fields strongly increased in the past years. Despite the numerous advantages of MS-based methods, one of the most challenging aspects is still related to the analysis of complex matrices for the detection of nontarget or unknown compounds. The creation of detailed libraries of compounds, including MS-based information such as accurate mass, 
isotopic patterns, and collision-induced fragmentation, is strongly demanded.

Innovations in ambient MS allowed the development of analytical methods characterized by high-throughput and minimal sample preparation, thus allowing the analysis of samples in their native ambient. However, an important feature to be discussed when ambient MS methods are used is related to the concentration of the detected compounds on the sample surface that might not represent the actual concentration in the whole sample, thus not matching the requirements of current legislation or official methods of analysis.

\section{Nomenclature}

$\begin{array}{ll}\text { CE: } & \text { Capillary electrophoresis } \\ \text { CNT: } & \text { Carbon nanotube } \\ \text { DART: } & \text { Direct analysis in real time } \\ \text { DESI: } & \text { Desorption electrospray ionization } \\ \text { DMPA: } & \text { N,N-dimethyl-p-phenylenediamine } \\ \text { EASI: } & \text { Easy ambient sonic-spray ionization } \\ \text { EC: } & \text { Electrochemical } \\ \text { EESI: } & \text { Extractive electrospray ionization } \\ \text { EI: } & \text { Electron ionization } \\ \text { ELDI: } & \text { Electrospray laser desorption ionization } \\ \text { ESI: } & \text { Electrospray ionization } \\ \text { ESTASI: } & \text { Electrostatic spray ionization } \\ \text { FA: } & \text { Fatty acid } \\ \text { FFA: } & \text { Free fatty acid } \\ \text { FI: } & \text { Field-induced } \\ \text { FT-ICR: } & \text { Fourier-transform ion cyclotron resonance } \\ \text { GC: } & \text { Gas chromatography } \\ \text { GC } \times \text { GC: } & \text { Two-dimensional gas chromatography } \\ \text { GO: } & \text { Graphene oxide } \\ \text { GOM: } & \text { Graphene oxide membrane } \\ \text { HMF: } & \text { Hydroxymethylfurfural } \\ \text { HILIC: } & \text { Hydrophilic interaction liquid } \\ \text { HPLC: } & \text { chromatography } \\ \text { HRMS: } & \text { High-performance liquid chromatography } \\ \text { LIT: } & \text { Linear ion trap } \\ \text { IT: } & \text { Ion trap } \\ \text { LAESI: } & \text { Laser ablation electrospray ionization } \\ \text { LC: } & \text { Liquid chromatography } \\ \text { LEI: } & \text { Liquid-electron ionization } \\ \text { LESA: } & \text { Liquid extraction surface analysis } \\ \text { LLE: } & \text { Liquid-liquid extraction } \\ \text { LS: } & \text { Liquid sample } \\ \text { LOQ: } & \text { Limit of quantitation } \\ \text { MALDI: } & \text { Matrix-assisted laser desorption/ionization } \\ \text { ME: } & \text { Matrix effect } \\ \text { MG: } & \text { Malachite green } \\ \text { MIP: } & \text { Molecularly imprinted polymer } \\ \text { MRL: } & \text { Maximum residue limit } \\ \text { Multiple reaction monitoring } \\ \text { Mass spectrometry } \\ \text { MSP } & \end{array}$

OTPAC: Octadecyldimethyl[3-(trimethoxysilyl)propyl] ammonium chloride

PAHs: $\quad$ Polycyclic aromatic hydrocarbons

PB: $\quad$ Paraffin barrier

PBDE: $\quad$ Polybrominated diphenyl ether

PCB: $\quad$ Polychlorobiphenyl

PDMS: $\quad$ Polydimethylsiloxane

PESI: $\quad$ Probe electrospray ionization

PFC: $\quad$ Perfluorinated compound

PMMA: $\quad$ Poly(methyl methacrylate)

PLA: $\quad$ Polylactate

PLE: $\quad$ Pressurized liquid extraction

PLLA: $\quad$ Poly-l-lactic acid

PSI: $\quad$ Paper spray ionization

PTFE: $\quad$ Polytetrafluoroethylene

Q: $\quad$ Quadrupole

QqQ: $\quad$ Triple quadrupole

QuEChERS: Quick easy cheap effective rugged safe

RSD: $\quad$ Relative standard deviation

SCWT: $\quad$ Surface-coated wooden tip

SFME: $\quad$ Slug-flow microextraction

SLE: Solid-liquid extraction

SMB: $\quad$ Supersonic molecular beam

SPE: $\quad$ Solid-phase extraction

SPME: $\quad$ Solid-phase microextraction

TLC: Thin-layer chromatography

TOF: $\quad$ Time of flight

TPPB: Tetraphenylphosphonium bromide

UPLC: Ultraperformance liquid chromatography

UTLC: Ultrathin-layer chromatography

WT: Wooden tip

ZV-PSI: Zero volt-paper spray ionization.

\section{Conflicts of Interest}

The authors declare that they have no conflicts of interest.

\section{References}

[1] T. Kind and O. Fiehn, "Advances in structure elucidation of small molecules using mass spectrometry," Bioanalytical Reviews, vol. 2, no. 1-4, pp. 23-60, 2010.

[2] R. Chen, J. Deng, L. Fang et al., "Recent applications of ambient ionization mass spectrometry in environmental analysis," Trends in Environmental Analytical Chemistry, vol. 15, pp. 1-11, 2017.

[3] M. Smoluch, P. Mielczarek, and J. Silberring, "Plasma-based ambient ionization mass spectrometry in bioanalytical sciences," Mass Spectrometry Reviews, vol. 35, no. 1, pp. 22-34, 2016.

[4] X. Ma and Z. Ouyang, "Ambient ionization and miniature mass spectrometry system for chemical and biological analysis," TrAC Trends in Analytical Chemistry, vol. 85, pp. 10-19, 2016.

[5] C. W. Klampfl and M. Himmelsbach, "Direct ionization methods in mass spectrometry: an overview," Analytica Chimica Acta, vol. 890, pp. 44-59, 2015.

[6] C. Y. Shi and C. H. Deng, "Recent advances in inorganic materials for LDI-MS analysis of small molecules," Analyst, vol. 141, no. 10, pp. 2816-2826, 2016. 
[7] B. B. Schneider, E. G. Nazarov, F. Londry, P. Vouros, and T. R. Covey, "Differential mobility spectrometry/mass spectrometry history, theory, design optimization, simulations, and applications," Mass Spectrometry Reviews, vol. 35, no. 6 , pp. 687-737, 2015.

[8] T. J. Kauppila, J. A. Syage, and T. Benter, "Recent developments in atmospheric pressure photoionization-mass spectrometry," Mass Spectrometry Reviews, vol. 36, no. 3, pp. 423-449, 2017.

[9] P. M. Peacock, W. J. Zhang, and S. Trimpin, "Advances in ionization for mass spectrometry," Analytical Chemistry, vol. 89, no. 1, pp. 372-388, 2017.

[10] Z. Takàts, J. M. Wiseman, B. Gologan, and R. G. Cooks, "Mass spectrometry sampling under ambient conditions with desorption electrospray ionization," Science, vol. 306, no. 5695, pp. 471-473, 2004.

[11] H. Wang, J. Liu, R. G. Cooks, and Z. Ouyang, "Paper spray for direct analysis of complex mixtures using mass spectrometry," Angewandte Chemie International Edition, vol. 49, no. 5, pp. 877-880, 2010.

[12] P. Nemes and A. Vertes, "Laser ablation electrospray ionization for atmospheric pressure, in vivo, and imaging mass spectrometry," Analytical Chemistry, vol. 79, no. 21, pp. 8098-8106, 2007.

[13] L. Li and K. A. Schug, "On- and off-line coupling of separation techniques to ambient ionization mass spectrometry," LCGC North America, vol. 9, no. 4, pp. 8-14, 2011.

[14] A. Herrmann, J. Rosen, D. Jansson, and K. E. Hellenas, "Evaluation of a generic multi-analyte method for detection of $>100$ representative compounds correlated to emergency events in 19 food types by ultrahigh-pressure liquid chromatography-tandem mass spectrometry," Journal of Chromatography A, vol. 1235, pp. 115-124, 2012.

[15] F. T. Peters, "Recent advances of liquid chromatography(tandem) mass spectrometry in clinical and forensic toxicology," Clinical Biochemistry, vol. 44, no. 1, pp. 54-65 2011.

[16] D. Remane, D. K. Wissenbach, and F. T. Peters, "Recent advances of liquid chromatography-(tandem) mass spectrometry in clinical and forensic toxicology-an update," Clinical Biochemistry, vol. 49, no. 13-14, pp. 1051-1071, 2016.

[17] F. T. Peters and D. Remane, "Aspects of matrix effects in applications of liquid chromatography-mass spectrometry to forensic and clinical toxicology-a review," Analytical and Bioanalytical Chemistry, vol. 403, no. 8, pp. 2155-2172, 2012.

[18] F. Gosetti, E. Mazzucco, D. Zampieri, and M. C. Gennaro, "Signal suppression/enhancement in high-performance liquid chromatography tandem mass spectrometry," Journal of Chromatography A, vol. 1217, no. 25, pp. 3929-3937, 2010.

[19] A. K. Malik, C. Blasco, and Y. Pico, "Liquid chromatographymass spectrometry in food safety," Journal of Chromatography $A$, vol. 1217, no. 25, pp. 4018-4040, 2010.

[20] A. Kaufmann, "The current role of high-resolution mass spectrometry in food analysis," Analytical and Bioanalytical Chemistry, vol. 403, no. 5, pp. 1233-1249, 2013.

[21] F. Hernandez, J. V. Sancho, M. Ibanez, E. Abad, T. Portoles, and L. Mattioli, "Current use of high-resolution mass spectrometry in the environmental sciences," Analytical and Bioanalytical Chemistry, vol. 403, no. 5, pp. 1251-1264, 2012.

[22] M. M. Gomez-Ramos, C. Ferrer, O. Malato, A. Aguera, and A. R. Fernandez-Alba, "Liquid chromatography-high-resolution mass spectrometry for pesticide residue analysis in fruit and vegetables: screening and quantitative studies," Journal of Chromatography A, vol. 1287, pp. 24-37, 2013.
[23] A. G. Marshall and C. L. Hendrickson, "High-resolution mass spectrometers," Annual Review of Analytical Chemistry, vol. 1, no. 1, pp. 579-599, 2008.

[24] Y. Fu, C. Zhao, X. Lu, and G. Xu, "Nontargeted screening of chemical contaminants and illegal additives in food based on liquid chromatography-high resolution mass spectrometry," TrAC Trends in Analytical Chemistry, vol. 96, pp. 89-98, 2017.

[25] M. Mattarozzi, M. Milioli, C. Cavalieri, F. Bianchi, and M. Careri, "Rapid desorption electrospray ionization-high resolution mass spectrometry method for the analysis of melamine migration from melamine tableware," Talanta, vol. 101, pp. 453-459, 2012.

[26] B. O. Crews, A. J. Pesce, R. West, H. Nguyen, and R. L. Fitzgerald, "Evaluation of high-resolution mass spectrometry for urine toxicology screening in a pain management setting," Journal of Analytical Toxicology, vol. 36, no. 9, pp. 601-607, 2012.

[27] F. Guale, S. Shahreza, J. P. Waltersheid, H. H. Chen, C. Arndt, and A. T. Kelly, "Validation of LC-TOF-MS screening for drugs, metabolites, and collateral compounds in forensic toxicology specimens," Journal of Analytical Toxicology, vol. 37, no. 1, pp. 17-24, 2013.

[28] F. W. McLafferty, Interpretation of Mass Spectra, University Science Books, Mill Valley, CA, USA, 1980.

[29] A. Cappiello, G. Famiglini, P. Palma, E. Pierini, V. Termopoli, and H. Trufelli, "Direct-EI in LC-MS: towards a universal detector for small-molecule applications," Mass Spectrometry Reviews, vol. 30, no. 6, pp. 1242-1255, 2011.

[30] P. Palma, G. Famiglini, H. Trufelli, E. Pierini, V. Termopoli, and A. Cappiello, "Electron ionization in LC-MS: recent developments and applications of the direct-EI LC-MS interface," Analytical and Bioanalytical Chemistry, vol. 399, no. 8, pp. 2683-2693, 2011.

[31] A. Cappiello, G. Famiglini, P. Palma, V. Termopoli, F. Capriotti, and N. Cellar, "Identification potential of directEI LC-MS interfacing in small-molecule applications," Journal of Separation Science, vol. 5, pp. 13-17, 2013.

[32] V. Termopoli, G. Famiglini, P. Palma, M. Piergiovanni, and A. Cappiello, "Atmospheric pressure vaporization mechanism for coupling a liquid phase with electron ionization mass spectrometry," Analytical Chemistry, vol. 89, no. 3, pp. 2049-2056, 2017.

[33] F. Rigano, A. Albergamo, D. Sciarrone, M. Beccaria, G. Purcaro, and L. Mondello, "Nano liquid chromatography directly coupled to electron ionization mass spectrometry for free fatty acid elucidation in mussel," Analytical Chemistry, vol. 88, no. 7, pp. 4021-4028, 2016.

[34] B. Seemann, T. Alon, S. Tsizin, A. B. Fialkov, and A. Amirav, "Electron ionization LC-MS with supersonic molecular beams-the new concept, benefits and applications," Journal of Mass Spectrometry, vol. 50, no. 11, pp. 1252-1263, 2015.

[35] G. A. Harris, A. S. Galhena, and F. M. Fernandez, "Ambient sampling/ionization mass spectrometry: applications and current trends," Analytical Chemistry, vol. 83, no. 12, pp. 4508-4538, 2011.

[36] M. Domin and R. Cody, Ambient Ionization Mass Spectrometry, Royal Society of Chemistry, Cambridge, UK, 2015.

[37] L. Magrini, G. Famiglini, P. Palma, V. Termopoli, and A. Cappiello, "Boosting the detection potential of liquid chromatography-electron ionization mass spectrometry using a ceramic coated ion source," Journal of the American Society for Mass Spectrometry, vol. 27, no. 1, pp. 153-160, 2016. 
[38] N. Riboni, L. Magrini, F. Bianchi, M. Careri, and A. Cappiello, "Sol-gel coated ion sources for liquid chromatography-direct electron ionization mass spectrometry," Analytica Chimica Acta, vol. 978, pp. 35-41, 2017.

[39] F. Bianchi, A. Gregori, G. Braun, C. Crescenzi, and M. Careri, "Micro-solid-phase extraction coupled to desorption electrospray ionization-high-resolution mass spectrometry for the analysis of explosives in soil," Analytical and Bioanalytical Chemistry, vol. 407, no. 3, pp. 931-938, 2014.

[40] N. L. Sanders, S. Kothari, G. Huang, G. Salazar, and R. G. Cooks, "Detection of explosives as negative ions directly from surfaces using a miniature mass spectrometer," Analytical Chemistry, vol. 82, no. 12, pp. 5313-5316, 2010.

[41] I. Cotte-Rodríguez, Z. Takats, N. Talaty, H. Chen, and R. G. Cooks, "Desorption electrospray ionization of explosives on surfaces: sensitivity and selectivity enhancement by reactive desorption electrospray ionization," Analytical Chemistry, vol. 77, no. 21, pp. 6755-6764, 2005.

[42] N. Talaty, C. C. Mulligan, D. R. Justes, A. U. Jackson, R. J. Noll, and R. G. Cooks, "Fabric analysis by ambient mass spectrometry for explosives and drugs," Analyst, vol. 133, no. 11, pp. 1532-1540, 2008.

[43] I. Cotte-Rodriguez, H. Hernandez-Soto, H. Chen, and R. G. Cooks, "In situ trace detection of peroxide explosives by desorption electrospray ionization and desorption atmospheric pressure chemical ionization," Analytical Chemistry, vol. 80, no. 5, pp. 1512-1519, 2008.

[44] J. M. Wiseman, D. R. Ifa, Y. Zhu et al., "Desorption electrospray ionization mass spectrometry: imaging drugs and metabolites in tissues," Proceedings of the National Academy of Sciences, vol. 105, no. 47, pp. 18120-18125, 2008.

[45] T. J. Kauppila, N. Talaty, T. Kuuranne, T. Kotiaho, R. Kostiainen, and R. G. Cooks, "Rapid analysis of metabolites and drugs of abuse from urine samples by desorption electrospray ionizationmass spectrometry," Analyst, vol. 132, no. 9, pp. 868-875, 2007.

[46] T. J. Kauppila, J. M. Wiseman, R. A. Ketola, T. Kotiaho, R. G. Cooks, and R. Kostiainen, "Desorption electrospray ionization mass spectrometry for the analysis of pharmaceuticals and metabolites," Rapid Communications in Mass Spectrometry, vol. 20, no. 3, pp. 387-392, 2006.

[47] Z. Lin, S. Zhang, M. Zhao, C. Yang, D. Chen, and X. Zhang, "Rapid screening of clenbuterol in urine samples by desorption electrospray ionization tandem mass spectrometry," Rapid Communications in Mass Spectrometry, vol. 22, no. 12, pp. 1882-1888, 2008.

[48] H. Chen, J. Zheng, X. Zhang, M. Luo, Z. Wang, and X. Qiao, "Surface desorption atmospheric pressure chemical ionization mass spectrometry for direct ambient sample analysis without toxic chemical contamination," Journal of Mass Spectrometry, vol. 42, no. 8, pp. 1045-1056, 2007.

[49] R. J. Fussell, D. Chan, and M. Sharman, "An assessment of atmospheric-pressure solids-analysis probes for the detection of chemicals in food," TrAC Trends in Analytical Chemistry, vol. 29, no. 11, pp. 1326-1335, 2010.

[50] C. Black, O. P. Chevallier, and C. T. Elliott, "The current and potential applications of ambient mass spectrometry in detecting food fraud," TrAC Trends in Analytical Chemistry, vol. 82, pp. 268-278, 2016.

[51] E. Hiyama, A. Ali, S. Amer et al., "Direct lipido-metabolomics of single floating cells for analysis of circulating tumor cells by live single-cell mass spectrometry," Analytical Sciences, vol. 31, no. 12, pp. 1215-1517, 2015.
[52] F. Chen, L. Lin, J. Zhang, Z. He, K. Uchiyama, and J. M. Lin, "Single-cell analysis using drop-on-demand inkjet printing and probe electrospray ionization mass spectrometry," Analytical Chemistry, vol. 88, no. 8, pp. 4354-4360, 2016.

[53] X. Gong, Y. Zhao, S. Cai et al., "Single cell analysis with probe ESI-mass spectrometry: detection of metabolites at cellular and subcellular levels," Analytical Chemistry, vol. 86, no. 8, pp. 3809-3816, 2014.

[54] Z. Takats, J. M. Wiseman, D. R. Ifa, and R. G. Cooks, "Desorption electrospray ionization (DESI) analysis of tryptic digests/peptides," Cold Spring Harbor Protocols, vol. 2008, no. 5, p. pdb.prot4993, 2008.

[55] S. P. Pasilis, V. Kertesz, G. J. Van Berkel, M. Schulz, and S. Schorcht, "Using HPTLC/DESI-MS for peptide identification in 1D separations of tryptic protein digests," Analytical and Bioanalytical Chemistry, vol. 391, no. 1, pp. 317-324, 2008.

[56] G. Parsiegla, B. Shrestha, F. Carriere, and A. Vertes, "Direct analysis of phycobilisomal antenna proteins and metabolites in small cyanobacterial populations by laser ablation electrospray ionization mass spectrometry," Analytical Chemistry, vol. 84, no. 1, pp. 34-38, 2012.

[57] Z. P. Yao, "Characterization of proteins by ambient mass spectrometry," Mass Spectrometry Reviews, vol. 31, no. 4, pp. 437-447, 2012.

[58] M. Morelato, A. Beavis, P. Kirkbride, and C. Roux, "Forensic applications of desorption electrospray ionisation mass spectrometry (DESI-MS)," Forensic Science International, vol. 226, no. 1-3, pp. 10-21, 2013.

[59] C. Ibáñez, V. García-Cañas, A. Valdés, and C. Simó, "Novel MS-based approaches and applications in food metabolomics," TrAC Trends in Analytical Chemistry, vol. 52, pp. 100-111, 2013.

[60] M. Castro-Puyana and M. Herrero, "Metabolomics approaches based on mass spectrometry for food safety, quality and traceability," TrAC Trends in Analytical Chemistry, vol. 52, pp. 74-87, 2013.

[61] P. Nemes and A. Vertes, "Ambient mass spectrometry for in vivo local analysis and in situ molecular tissue imaging," TrAC Trends in Analytical Chemistry, vol. 34, pp. 22-34, 2012.

[62] E. R. St John, M. Rossi, P. Pruski, A. Darzi, and Z. Takats, "Intraoperative tissue identification by mass spectrometric technologies," TrAC Trends in Analytical Chemistry, vol. 85, pp. 2-9, 2016.

[63] Y. Yang, Y. Huang, J. Wu, N. Liu, J. Deng, and T. Luan, "Single-cell analysis by ambient mass spectrometry," TrAC Trends in Analytical Chemistry, vol. 90, pp. 14-26, 2017.

[64] A. A. Lubin, D. Cabooter, P. Augustijns, and F. Cuyckens, "One drop chemical derivatization - DESI-MS analysis for metabolite structure identification," Journal of Mass Spectrometry, vol. 50, no. 7, pp. 871-878, 2015.

[65] T. A. Brown, H. Chen, and R. N. Zare, "Identification of fleeting electrochemical reaction intermediates using desorption electrospray ionization mass spectrometry," Journal of the American Chemical Society, vol. 137, no. 23, pp. 7274-7277, 2015.

[66] W. D. Looi, B. Brown, L. Chamand, and A. Brajter-Toth, "Merits of online electrochemistry liquid sample desorption electrospray ionization mass spectrometry (EC/LS DESI MS)," Analytical and Bioanalytical Chemistry, vol. 408, no. 9, pp. 2227-2238, 2016.

[67] Y. Cai, D. Adams, and H. Chen, "A new splitting method for both analytical and preparative LC/MS," Journal of the 
American Society for Mass Spectrometry, vol. 25, no. 2, pp. 286-292, 2014.

[68] Y. Cai, Y. Liu, R. Helmy, and H. Chen, "Coupling of ultrafast LC with mass spectrometry by DESI," Journal of The American Society for Mass Spectrometry, vol. 25, no. 10, pp. 1820-1823, 2014.

[69] Y. Ren, M. N. McLuckey, J. Liu, and Z. Ouyang, "Direct mass spectrometry analysis of biofluid samples using slug-flow microextraction nano-electrospray ionization," Angewandte Chemie International Edition, vol. 53, no. 51, pp. 1412414127, 2014.

[70] J. Cain, A. Laskin, M. R. Kholghy, M. J. Thomson, and H. Wang, "Molecular characterization of organic content of soot along the centerline of a coflow diffusion flame," Physical Chemistry Chemical Physics, vol. 16, no. 47, pp. 25862-25875, 2014.

[71] S. Tao, X. Lu, N. Levac et al., "Molecular characterization of organosulfates in organic aerosols from Shanghai and Los Angeles urban areas by nanospray-desorption electrospray ionization high-resolution mass spectrometry," Environmental Science \& Technology, vol. 48, no. 18, pp. 1099311001, 2014.

[72] E. J. Boone, A. Laskin, J. Laskin et al., “Aqueous processing of atmospheric organic particles in cloud water collected via aircraft sampling," Environmental Science \& Technology, vol. 49, no. 14, pp. 8523-8530, 2015.

[73] C. Cardoso-Palacios and I. Lanekoff, "Direct analysis of pharmaceutical drugs using nano-DESI MS," Journal of Analytical Methods in Chemistry, vol. 2016, Article ID 3591908, 6 pages, 2016.

[74] J. Watrous, P. Roach, B. Heath, T. Alexandrov, J. Laskin, and P. C. Dorrestein, "Metabolic profiling directly from the Petri dish using nanospray desorption electrospray ionization imaging mass spectrometry," Analytical Chemistry, vol. 85, no. 21, pp. 10385-10391, 2013.

[75] H. M. Bergman and I. Lanekoff, "Profiling and quantifying endogenous molecules in single cells using nano-DESI MS," Analyst, vol. 142, no. 19, pp. 3639-3647, 2017.

[76] H. M. Bergman, E. Lundin, M. Andersson, and I. Lanekoff, "Quantitative mass spectrometry imaging of small-molecule neurotransmitters in rat brain tissue sections using nanospray desorption electrospray ionization," Analyst, vol. 141, no. 12 , pp. 3686-3695, 2016.

[77] C. C. Hsu, P. T. Chou, and R. N. Zare, "Imaging of proteins in tissue samples using nanospray desorption electrospray ionization mass spectrometry," Analytical Chemistry, vol. 87, no. 22, pp. 11171-11175, 2015.

[78] D. Duncan, H. M. Bergman, and I. Lanekoff, "A pneumatically assisted nanospray desorption electrospray ionization source for increased solvent versatility and enhanced metabolite detection from tissue," Analyst, vol. 142, no. 18, pp. 3424-3431, 2017.

[79] H. W. Chen, A. Venter, and R. G. Cooks, "Extractive electrospray ionization for direct analysis of undiluted urine, milk and other complex mixtures without sample preparation," Chemical Communications, vol. 42, no. 19, pp. 2042-2044, 2006.

[80] R. Wang, A. J. Gröhn, L. Zhu et al., "On the mechanism of extractive electrospray ionization (EESI) in the dual-spray configuration," Analytical and Bioanalytical Chemistry, vol. 402, no. 8, pp. 2633-2643, 2012.

[81] P. J. Gallimore and M. Kalberer, "Characterizing an extractive electrospray ionization (EESI) source for the online mass spectrometry analysis of organic aerosols,"
Environmental Science \& Technology, vol. 47, no. 13, pp. 7324-7331, 2013.

[82] G. K. Koyanagi, V. Blagojevic, and D. K. Bohme, "Applications of extractive electrospray ionization (EESI) in analytical chemistry," International Journal of Mass Spectrometry, vol. 379, pp. 146-150, 2015.

[83] M. Deng, T. Yu, H. Luo, T. Zhu, X. Huang, and L. Luo, "Direct detection of multiple pesticides in honey by neutral desorption-extractive electrospray ionization mass spectrometry," International Journal of Mass Spectrometry, vol. 422, pp. 111-118, 2017.

[84] N. Xu, Z. Q. Zhu, S. P. Yang et al., "Direct detection of amino acids using extractive electrospray ionization tandem mass spectrometry," Chinese Journal of Analytical Chemistry, vol. 41, no. 4, p. 523, 2013.

[85] X. Li, X. Fang, Z. Yu et al., "Direct quantification of creatinine in human urine by using isotope dilution extractive electrospray ionization tandem mass spectrometry," Analytica Chimica Acta, vol. 748, pp. 53-57, 2012.

[86] R. S. Jacobson, R. L. Thurston, B. Shrestha, and A. Vertes, "In situ analysis of small populations of adherent mammalian cells using laser ablation electrospray ionization mass spectrometry in transmission geometry," Analytica Chimica Acta, vol. 87, no. 24, pp. 12130-12136, 2015.

[87] L. R. Compton, B. Reschke, J. Friend, M. Powell, and A. Vertes, "Remote laser ablation electrospray ionization mass spectrometry for non-proximate analysis of biological tissues," Rapid Communications in Mass Spectrometry, vol. 29, no. 1, pp. 67-73, 2015.

[88] L. C. Duarte, T. C. de Carvalho, E. O. Lobo-Júnior, P. V. Abdelnur, B. G. Vaza, and W. K. T. Coltro, "3D printing of microfluidic devices for paper-assisted direct spray ionization mass spectrometry," Analytical Methods, vol. 8, no. 3, pp. 496-503, 2016.

[89] G. I. J. Salentijn, H. P. Permentier, and E. Verpoorte, “3Dprinted paper spray ionization cartridge with fast wetting and continuous solvent supply features," Analytical Chemistry, vol. 86, no. 23, pp. 11657-11665, 2014.

[90] L. Shen, J. Zhang, Q. Yang, N. E. Manicke, and Z. Ouyang, "High throughput paper spray mass spectrometry analysis," Clinica Chimica Acta, vol. 420, pp. 28-33, 2013.

[91] J. Deng, W. Wang, Y. Yang et al., "Slug-flow microextraction coupled with paper spray mass spectrometry for rapid analysis of complex samples," Analytica Chimica Acta, vol. 940, pp. 143-149, 2016.

[92] C. Zhang and N. E. Manicke, "Development of a paper spray mass spectrometry cartridge with integrated solid phase extraction for bioanalysis," Analytical Chemistry, vol. 87, no. 12, pp. 6212-6219, 2015.

[93] B. Yang, F. Wang, W. Deng et al., "Wooden-tip electrospray ionization mass spectrometry for trace analysis of toxic and hazardous compounds in food samples," Analytical Methods, vol. 7, no. 14, pp. 5886-5890, 2015.

[94] G. Z. Xin, B. Hu, Z. Q. Shi et al., "Rapid identification of plant materials by wooden-tip electrospray ionization mass spectrometry and a strategy to differentiate the bulbs of Fritillaria," Analytica Chimica Acta, vol. 820, pp. 84-91, 2014.

[95] Y. Yang and J. Deng, "Internal standard mass spectrum fingerprint: a novel strategy for rapid assessing the quality of Shuang-Huang-Lian oral liquid using wooden-tip electrospray ionization mass spectrometry," Analytica Chimica Acta, vol. 837, pp. 83-92, 2014. 
[96] H. K. Chen, C. H. Lin, J. T. Liu, and C. H. Lin, "Electrospray ionization using a bamboo pen nib," International Journal of Mass Spectrometry, vol. 356, pp. 37-40, 2013.

[97] B. Hu and Z. P. Yao, "Detection of native proteins using solid-substrate electrospray ionization mass spectrometry with nonpolar solvents," Analytica Chimica Acta, vol. 1004, pp. 51-57, 2017.

[98] Y. Yang, J. Deng, and Z. P. Yao, "Field-induced wooden-tip electrospray ionization mass spectrometry for highthroughput analysis of herbal medicines," Analytica Chimica Acta, vol. 887, pp. 127-137, 2015.

[99] Y. W. Liou, K. Y. Chang, and C. H. Lin, "Sampling and profiling caffeine and its metabolites from an eyelid using a watercolor pen based on electrospray ionization/mass spectrometry," International Journal of Mass Spectrometry, vol. 422, pp. 51-55, 2017.

[100] N. Pan, W. Rao, N. R. Kothapalli, R. Liu, A. W. G. Burgett, and Z. Yang, "The single-probe: a miniaturized multifunctional device for single cell mass spectrometry analysis," Analytical Chemistry, vol. 86, no. 19, pp. 9376-9380, 2014.

[101] C. Shiea, Y. L. Huang, S. C. Cheng, Y. L. Chen, and J. Shiea, "Determination of elemental composition of metals using ambient organic mass spectrometry," Analytica Chimica Acta, vol. 968, pp. 50-57, 2017.

[102] L. Qiao, R. Sartor, N. Gasilova et al., "Electrostatic-spray ionization mass spectrometry," Analytical Chemistry, vol. 84, no. 17, pp. 7422-7430, 2012.

[103] Z. Takats, J. M. Wiseman, and R. G. Cooks, “Ambient mass spectrometry using desorption electrospray ionization (DESI): instrumentation, mechanisms and applications in forensics, chemistry, and biology," Journal of Mass Spectrometry, vol. 40, no. 10, pp. 1261-1275, 2005.

[104] A. Penna, M. Careri, N. D. Spencer, and A. Rossi, "Effects of tailored surface chemistry on desorption electrospray ionization mass spectrometry: a surface-analytical study by XPS and AFM," Journal of the American Society for Mass Spectrometry, vol. 26, no. 8, pp. 1311-1319, 2015.

[105] L. Elviri, R. Foresti, A. Bianchera, M. Silvestri, and R. Bettini, "3D-printed polylactic acid supports for enhanced ionization efficiency in desorption electrospray mass spectrometry analysis of liquid and gel samples," Talanta, vol. 155, pp. 321-328, 2016.

[106] M. Montowska, W. Rao, M. R. Alexander, G. A. Tucker, and D. A. Barrett, "Tryptic digestion coupled with ambient desorption electrospray ionization and liquid extraction surface analysis mass spectrometry enabling identification of skeletal muscle proteins in mixtures and distinguishing between beef, pork, horse, chicken, and turkey meat," Analytical Chemistry, vol. 86, no. 9, pp. 4479-4487, 2014.

[107] M. T. Dulay, L. S. Eberlin, and R. N. Zare, "Protein analysis by ambient ionization mass spectrometry using trypsinimmobilized organosiloxane polymer surfaces," Analytical Chemistry, vol. 87, no. 24, pp. 12324-12330, 2015.

[108] S. Cheng, J. Wang, Y. Cai, J. A. Loo, and H. Chen, "Enhancing performance of liquid sample desorption electrospray ionization mass spectrometry using trap and capillary columns," International Journal of Mass Spectrometry, vol. 392, pp. 73-79, 2015.

[109] S. S. Kanyal, T. T. Häbe, C. V. Cushman et al., "Microfabrication, separations, and detection by mass spectrometry on ultrathin-layer chromatography plates prepared via the low-pressure chemical vapor deposition of silicon nitride onto carbon nanotube templates," Journal of Chromatography A, vol. 1404, pp. 115-123, 2015.
[110] K. J. Ewing, D. Gibson, J. Sanghera, and F. Miklos, "Desorption electrospray ionization-mass spectrometric analysis of low vapor pressure chemical particulates collected from a surface," Analytica Chimica Acta, vol. 853, pp. 368-374, 2015.

[111] R. G. Hemalatha, M. A. Ganayee, and T. Pradeep, "Electrospun nanofiber mats as "smart surfaces" for desorption electrospray ionization mass spectrometry (DESI MS)-based analysis and imprint imaging," Analytical Chemistry, vol. 88, no. 11, pp. 5710-5717, 2016.

[112] C. H. Lin, W. C. Liao, H. K. Chen, and T. Y. Kuo, "Paper spray-MS for bioanalysis," Bioanalysis, vol. 6, no. 2, pp. 1-10, 2014.

[113] Q. Yang, H. Wang, J. D. Maas et al., "Paper spray ionization devices for direct, biomedical analysis using mass spectrometry," International Journal of Mass Spectrometry, vol. 312, pp. 201-207, 2012.

[114] H. Evard, A. Kruve, R. Lõhmus, and I. Leito, "Paper spray ionization mass spectrometry: study of a method for fastscreening analysis of pesticides in fruits and vegetables," Journal of Food Composition and Analysis, vol. 41, pp. 221225, 2015.

[115] Z. P. Zhang, X. N. Liu, and Y. J. Zheng, "Ambient ionizationpaper spray ionization and its application," Chinese Journal of Analytical Chemistry, vol. 42, no. 1, pp. 145-152, 2014.

[116] P. H. Lai, P. C. Chen, Y. W. Liao, J. T. Liu, C. C. Chen, and C. H. Lin, "Comparison of gampi paper and nanofibers to chromatography paper used in paper spray-mass spectrometry," International Journal of Mass Spectrometry, vol. 375, pp. 14-17, 2015.

[117] T. C. Colletes, P. T. Garcia, R. B. Campanha et al., "A new insert sample approach to paper spray mass spectrometry: a paper substrate with paraffin barriers," Analyst, vol. 141, no. 5, pp. 1707-1713, 2016.

[118] Z. Zhang, W. Xu, N. E. Manicke, R. G. Cooks, and Z. Ouyang, "Silica coated paper substrate for paper-spray analysis of therapeutic drugs in dried blood spots," Analytical Chemistry, vol. 84, no. 2, pp. 931-938, 2012.

[119] R. Narayanan, D. Sarkar, R. G. Cooks, and T. Pradeep, "Molecular ionization from carbon nanotube paper," Angewandte Chemie International Edition, vol. 53, no. 23, pp. 5936-5940, 2014.

[120] S. C. Wei, S. Fan, C. W. Lien et al., "Graphene oxide membrane as an efficient extraction and ionization substrate for spray-mass spectrometric analysis of malachite green and its metabolite in fish samples," Analytica Chimica Acta, vol. 1003, pp. 42-48, 2018.

[121] J. Liu, Y. He, S. Chen, M. Ma, S. Yao, and B. Chen, "New urea-modified paper substrate for enhanced analytical performance of negative ion mode paper spray mass spectrometry," Talanta, vol. 166, pp. 306-314, 2017.

[122] I. Pereira, M. F. Rodrigues, A. R. Chaves, and B. G. Vaz, "Molecularly imprinted polymer (MIP) membrane assisted direct spray ionization mass spectrometry for agrochemicals screening in foodstuffs," Talanta, vol. 178, pp. 507-514, 2018.

[123] B. J. Bills and N. E. Manicke, "Development of a prototype blood fractionation cartridge for plasma analysis by paper spray mass spectrometry," Clinical Mass Spectrometry, vol. 2, pp. 18-24, 2016.

[124] M. Wleklinski, Y. Li, S. Bag et al., "Zero volt paper spray ionization and its mechanism," Analytical Chemistry, vol. 87, no. 13, pp. 6786-6793, 2015.

[125] J. Deng, Y. Yang, L. Fang, L. Lin, H. Zhou, and T. Luan, "Coupling solid-phase microextraction with ambient mass spectrometry using surface coated wooden-tip probe for 
rapid analysis of ultra trace perfluorinated compounds in complex samples," Analytical Chemistry, vol. 86, no. 22, pp. 11159-11166, 2014.

[126] J. Deng, T. Yu, Y. Yao et al., "Surface-coated wooden-tip electrospray ionization mass spectrometry for determination of trace fluoroquinolone and macrolide antibiotics in water," Analytica Chimica Acta, vol. 954, pp. 52-59, 2017.

[127] Y. Huang, Y. Ma, H. Hu et al., "Rapid and sensitive detection of trace malachite green and its metabolite in aquatic products using molecularly imprinted polymer-coated wooden-tip electrospray ionization mass spectrometry," RSC Advances, vol. 7, no. 82, pp. 52091-52100, 2017.

[128] B. Hu, P. K. So, and Z. P. Yao, "Electrospray ionization with aluminum foil: a versatile mass spectrometric technique," Analytica Chimica Acta, vol. 817, pp. 1-8, 2014.

[129] X. Zhong, L. Qiao, B. Liu, and H. H. Girault, "Ambient in situ analysis and imaging of both hydrophilic and hydrophobic thin layer chromatography plates by electrostatic spray ionization mass spectrometry," RSC Advances, vol. 5, no. 92, pp. 75395-75402, 2015.

[130] V. Garcia-Canas, M. Herrero, E. Ibanez, and A. Cifuentes, "Food analysis: present, future, and foodomics," Analytical Chemistry, vol. 84, no. 23, pp. 10150-10159, 2012.

[131] M. Herrero, C. Simo, V. Garcia-Canas, E. Ibanez, and A. Cifuentes, "Foodomics: MS-based strategies in modern food science and nutrition," Mass Spectrometry Reviews, vol. 31, no. 1, pp. 49-69, 2012.

[132] F. Cacciola, P. Donato, M. Beccaria, P. Dugo, and L. Mondello, "Advances in LC-MS for food analysis," LC GC Europe, vol. 25, no. 5, pp. 15-24, 2012.

[133] European Union Commission Decision 2002/657/EC, 2002.

[134] G. C. R. M. Andrade, S. H. Monteiro, J. G. Francisco, L. A. Figueiredo, R. G. Botelho, and V. L. Tornisielo, "Liquid chromatography-electrospray ionization tandem mass spectrometry and dynamic multiple reaction monitoring method for determining multiple pesticide residues in tomato," Food Chemistry, vol. 175, pp. 57-65, 2015.

[135] O. Golge and B. Kabak, "Evaluation of QuEChERS sample preparation and liquid chromatography-triple-quadrupole mass spectrometry method for the determination of 109 pesticide residues in tomatoes," Food Chemistry, vol. 176, pp. 319-332, 2015.

[136] F. Diniz Madureira, F. A. da Silva Oliveira, W. R. de Souza, A. P. Pontelo, M. L. Gonçalves de Oliveira, and G. Silva, "A multi-residue method for the determination of 90 pesticides in matrices with a high water content by LC-MS/MS without clean-up," Food Additives \& Contaminants: Part A, vol. 29, no. 4, pp. 665-678, 2012.

[137] A. Garrido-Frenich, M. M. Martín Fernández, L. Díaz Moreno, J. L. Martínez-Vidal, and N. López-Gutiérrez, "Multiresidue pesticide analysis of tuber and root commodities by QuEChERS extraction and UPLC coupled to tandem MS," Journal of AOAC International, vol. 95, no. 5, pp. 1319-1330, 2012.

[138] O. Golge and B. Kabak, "Determination of 115 pesticide residues in oranges by high-performance liquid chromatography-triplequadrupole mass spectrometry in combination with QuEChERS method," Journal of Food Composition and Analysis, vol. 41, pp. 86-97, 2015.

[139] J. Cho, J. Lee, C. U. Lim, and J. Ahn, "Quantification of pesticides in food crops using QuEChERS approaches and GCeMS/MS," Food Additives \& Contaminants: Part A, vol. 33, no. 12, pp. 1803-1816, 2016.
[140] S. S. Shida, S. Nemoto, and R. Matsuda, "Simultaneous determination of acidic pesticides in vegetables and fruits by liquid chromatography-tandem mass spectrometry," Journal of Environmental Science and Health, Part B, vol. 50, no. 3, pp. 151-162, 2015.

[141] C. Rasche, B. Fournes, U. Dirks, and K. Speer, "Multi-residue pesticide analysis (gas chromatography-tandem mass spectrometry detection)-improvement of the quick, easy, cheap, effective, rugged, and safe method for dried fruits and fat-rich cereals-benefit and limit of a standardized apple purée calibration (screening)," Journal of Chromatography A, vol. 1403, pp. 21-31, 2015.

[142] L. Han, J. Matarrita, Y. Sapozhnikova, and S. J. Lehotay, "Evaluation of a recent product to remove lipids and other matrix co-extractives in the analysis of pesticide residues and environmental contaminants in foods," Journal of Chromatography A, vol. 1449, pp. 17-29, 2016.

[143] B. D. Morris and R. B. Schriner, "Development of an automated column solid-phase extraction cleanup of QuEChERS extracts, using a zirconia-based sorbent, for pesticide residue analyses by LC-MS/MS," Journal of Agricultural and Food Chemistry, vol. 63, no. 21, pp. 5107-5119, 2015.

[144] G. Ramadan, M. Al Jabir, N. Alabdulmalik, and A. Mohammed, "Validation of a method for the determination of 120 pesticide residues in apples and cucumbers by LC-MS/MS," Drug Testing and Analysis, vol. 8, no. 5-6, pp. 498-510, 2016.

[145] M. A. Zhao, Y. N. Feng, Y. Z. Zhu, and J. H. Kim, "Multiresidue method for determination of 238 pesticides in Chinese cabbage and cucumber by liquid chromatographytandem mass spectrometry: comparison of different purification procedures," Journal of Agricultural and Food Chemistry, vol. 62, no. 47, pp. 11449-11456, 2014.

[146] N. Fleury-Filho, C. A. Nascimento, E. O. Faria, A. R. Crunivel, and J. M. Oliveira, "Within laboratory validation of a multiresidue method for the analysis of 98 pesticides in mango by LC tandem MS," Food Additives \& Contaminants: Part A, vol. 29, no. 4, pp. 641-656, 2012.

[147] T. Cajka, C. Sandy, V. Bachavolva et al., "Streamlining sample preparation and GC tandem MS analysis of multiple pesticide residues in tea," Analytica Chimica Acta, vol. 743, pp. 51-60, 2012.

[148] V. Havolt, S. Goscinny, and M. Deridder, "A simple multiresidue method for the determination of pesticides in fruits and vegetables using a methanolic extraction and ultra-highperformance liquid chromatography-tandem mass spectrometry: optimization and extension of scope," Journal of Chromatography A, vol. 1384, pp. 53-66, 2015.

[149] S. Walorczyk and D. Drozdzynski, "Improvement and extension to new analytes of a multi-residue method for the determination of pesticides in cereals and dry animal feed using gas chromatography-tandem quadrupole mass spectrometry revisited," Journal of Chromatography A, vol. 1251, pp. 219-231, 2012.

[150] O. Lacina, M. Zachariasova, J. Urbavolva, M. Vaclavikova, T. Cajka, and J. Hajslova, "Critical assessment of extraction methods for the simultaneous determination of pesticide residues and mycotoxins in fruits, cereals, spices and oil seeds employing UPLC tandem MS," Journal of Chromatography A, vol. 1262, pp. 8-18, 2012.

[151] J. Wang, W. Chow, and W. Cheung, "Application of a tandem mass spectrometer and core-shell particle column for the determination of 151 pesticides in grains," Journal of Agricultural and Food Chemistry, vol. 59, no. 16, pp. 85898608, 2011. 
[152] Z. He, L. Wang, Y. Peng, M. Luo, W. Wang, and X. Liu, "Multiresidue analysis of over 200 pesticides in cereals using a QuEChERS and gas chromatography-tandem mass spectrometry-based method," Food Chemistry, vol. 169, pp. 372-380, 2015.

[153] A. Paleníkova, G. Martínez-Domínguez, F. J. Arrebola, R. Romero-Gonzalez, S. Hrouzkova, and A. Garrido Frenich, "Multifamily determination of pesticide residues in soyabased nutraceutical products by GC/MS-MS," Food Chemistry, vol. 173, pp. 796-807, 2015.

[154] S. Chawla, H. K. Patel, K. M. Vaghela et al., "Development and validation of multi residue analytical method in cotton and groundnut oil for 87 pesticides using low temperature and dispersive cleanup on gas chromatography and liquid chromatography-tandem mass spectrometry," Analytical and Bioanalytical Chemistry, vol. 408, no. 3, pp. 983-997, 2016.

[155] D. L. Christodoulou, P. Kanari, P. Hadjiloizou, and P. Constantivolu, "Pesticide residues analysis in wine by liquid chromatographytandem mass spectrometry and using ethyl acetate extraction method: validation and pilot survey in real samples," Journal of Wine Research, vol. 26, no. 2, pp. 81-98, 2015.

[156] G. R. Chang, H. S. Chen, and F. Y. Lin, "Analysis of banned veterinary drugs and herbicide residues in shellfish by liquid chromatography-tandem mass spectrometry (LC/MS/MS) and gas chromatography-tandem mass spectrometry (GC/MS/MS)," Marine Pollution Bulletin, vol. 113, no. 1-2, pp. 579-584, 2016.

[157] M. E. Dasenaki, C. S. Michali, and N. S. Thomaidis, "Analysis of 76 veterinary pharmaceuticals from 13 classes including aminoglycosides in bovine muscle by hydrophilic interaction liquid chromatography-tandem mass spectrometry," Journal of Chromatography A, vol. 1452, pp. 67-80, 2016.

[158] D. Chen, J. Yu, Y. Tao et al., "Qualitative screening of veterinary anti-microbial agents in tissues, milk, and eggs of food-producing animals using liquid chromatography coupled with tandem mass spectrometry," Journal of Chromatography B, vol. 1017-1018, pp. 82-88, 2016.

[159] M. Aznar, A. Rodriguez-Lafuente, P. Alfaro, and C. Nerin, "UPLC-Q-TOF-MS analysis of non-volatile migrants from new active packaging materials," Analytical and Bioanalytical Chemistry, vol. 404, no. 6-7, pp. 1945-1957, 2012.

[160] B. Skribic, J. Zivancev, and M. Godula, "Multimycotoxin analysis of crude extracts of nuts with ultra-high performance liquid chromatography/tandem mass spectrometry," Journal of Food Composition and Analysis, vol. 34, no. 2, pp. 171-177, 2014.

[161] M. Ludovici, C. Ialongo, M. Reverberi, M. Beccaccioli, M. Scarpari, and V. Scala, "Quantitative profiling of oxylipins through comprehensive LC-MS/MS analysis of Fusarium verticillioides and maize kernels," Food Additives \& Contaminants: Part A, vol. 31, no. 12, pp. 2026-2033, 2014.

[162] M. García-Altares, A. Casavolva, V. Bane, J. Diogene, A. Furey, and P. de la Iglesia, "Confirmation of pinnatoxins and spirolides in shellfish and passive samplers from Catalonia (Spain) by liquid chromatography coupled with triple quadrupole and high-resolution hybrid tandem mass spectrometry," Marine Drugs, vol. 12, no. 6, pp. 3706-3732, 2014.

[163] Y. Rodriguez-Carrasco, J. Manes, H. Berrada, and C. Juan, "Development and validation of a LC-ESI-MS/MS method for the determination of alternaria toxins alternariol, alternariol methyl-ether and tentoxin in tomato and tomato based products," Toxins, vol. 8, no. 11, p. 328, 2016.
[164] Y. Rodriguez-Carrasco, M. Fattore, S. Albrizio, H. Berrada, and J. Manes, "Occurrence of Fusarium mycotoxins and their dietary intake through beer consumption by the European population," Food Chemistry, vol. 178, pp. 149-155, 2015.

[165] C. Juan, J. Mañes, A. Raiola, and A. Ritieni, "Evaluation of beauvericin and enniatins in Italian cereal products and multicereal food by liquid chromatography coupled to triple quadrupole mass spectrometry," Food Chemistry, vol. 140, no. 4, pp. 755-762, 2013.

[166] K. Zhang, J. W. Wong, P. Yang et al., "Protocol for an electrospray ionization tandem mass spectral product ion library: development and application for identification of 240 pesticides in foods," Analytical Chemistry, vol. 84, no. 13, pp. 5677-5684, 2012.

[167] M. I. Cervera, T. Portoles, E. Pitarch, J. Beltran, and F. Hernandez, "Application of gas chromatography time-offlight mass spectrometry for target and non-target analysis of pesticide residues in fruits and vegetables," Journal of Chromatography A, vol. 1244, pp. 168-177, 2012.

[168] F. Lambertini, V. Di Lallo, D. Catellani, M. Mattarozzi, M. Careri, and M. Suman, "Reliable liquid chromatographymass spectrometry method for investigation of primary aromatic amines migration from food packaging and during industrial curing of multilayer plastic laminates," Journal of Mass Spectrometry, vol. 49, no. 9, pp. 870-877, 2014.

[169] C. Planche, J. Ratel, F. Mercier, P. Blinet, L. Debrauwer, and E. Engel, "Assessment of comprehensive two-dimensional gas chromatography-time-of-flight mass spectrometry based methods for investigating 206 dioxin-like micropollutants in animal-derived food matrices," Journal of Chromatography A, vol. 1392, pp. 74-81, 2015.

[170] X. Wang, P. Li, W. Zhang et al., "Screening for pesticide residues in oil seeds using solid-phase dispersion extraction and comprehensive two-dimensional gas chromatography time-of-flight mass spectrometry," Journal of Separation Science, vol. 35, no. 13, pp. 1634-1643, 2012.

[171] K. Kalachova, J. Pulkrabova, T. Cajka, L. Drabova, and J. Hajslova, "Implementation of comprehensive twodimensional GC-time-of-flight-MS for the simultaneous determination of halogenated contaminants and polycyclic aromatic hydrocarbons in fish," Analytical and Bioanalytical Chemistry, vol. 403, no. 10, pp. 2813-2824, 2012.

[172] M. L. Gomez-Perez, P. Plaza-Bolavols, R. Romero-Gonzalez, J. L. Martinez-Vidal, and A. Garrido-Frenich, "Comprehensive qualitative and quantitative determination of pesticides and veterinary drugs in honey using liquid chromatography-Orbitrap high resolution mass spectrometry," Journal of Chromatography A, vol. 1248, pp. 130-138, 2012.

[173] P. Perez-Ortega, F. J. Lara-Ortega, J. F. García-Reyes, B. GilbertLopez, M. Trojavolwicz, and A. Molina-Díaz, "A feasibility study of UHPLC-HRMS accurate-mass screening methods for multiclass testing of organic contaminants in food," Talanta, vol. 160, pp. 704-712, 2016.

[174] H. G. J. Mol, P. Zomer, and M. de Koning, "Qualitative aspects and validation of a screening method for pesticides in vegetables and fruits based on liquid chromatography coupled to full scan high resolution (Orbitrap) mass spectrometry," Analytical and Bioanalytical Chemistry, vol. 403, no. 10, pp. 2891-2908, 2012.

[175] J. Rubert, K. J. James, J. Manes, and C. Soler, “Applicability of hybrid linear ion trap-high resolution mass spectrometry and quadrupole-linear ion trap-mass spectrometry for 
mycotoxin analysis in baby food," Journal of Chromatography A, vol. 1223, pp. 84-92, 2012.

[176] D. G. Beach, C. M. Walsh, and P. McCarron, "Highthroughput quantitative analysis of domoic acid directly from mussel tissue using laser ablation electrospray ionization-tandem mass spectrometry," Toxicon, vol. 92, pp. 75-80, 2014.

[177] M. Guijarro-Díez, L. Volzal, M. L. Marina, and A. L. Crego, "Metabolomic fingerprinting of saffron by LC/MS: novel authenticity markers," Analytical and Bioanalytical Chemistry, vol. 407, no. 23, pp. 7197-7213, 2015.

[178] L. Millán, M. C. Sampedro, A. Sanchez et al., "Liquid chromatography-quadrupole time of flight tandem mass spectrometry-based targeted metabolomic study for varietal discrimination of grapes according to plant sterols content," Journal of Chromatography A, vol. 1454, pp. 67-77, 2016.

[179] M. Arbulu, M. C. Sampedro, A. Gomez-Caballero, M. A. Goicolea, and R. J. Barrio, "Untargeted metabolomic analysis using liquid chromatography quadrupole time-of-flight mass spectrometry for non-volatile profiling of wines," Analytica Chimica Acta, vol. 858, pp. 32-41, 2015.

[180] J. Rubert, O. Lacina, M. Zachariasova, and J. Hajslova, "Saffron authentication based on liquid chromatography high resolution tandem mass spectrometry and multivariate data analysis," Food Chemistry, vol. 204, pp. 201-209, 2016.

[181] A. Kårlund, U. Moor, G. McDougall, M. Lehtonen, R. O. Karjalainen, and K. Hanhineva, "Metabolic profiling discriminates between strawberry (Fragaria $\times$ ananassa Duch.) cultivars grown in Finland or Estonia," Food Research International, vol. 89, pp. 647-653, 2016.

[182] Z. Jandric, D. Roberts, M. N. Rathor, A. Abrahim, M. Islam, and A. Cannavan, "Assessment of fruit juice authenticity using UPLC-QToF MS: a metabolomics approach," Food Chemistry, vol. 148, pp. 7-17, 2014.

[183] Z. Jandric, M. Islam, D. K. Singh, and A. Cannavan, "Authentication of Indian citrus fruit/fruit juices by untargeted and targeted metabolomics," Food Control, vol. 72, pp. 181-188, 2017.

[184] S. Jin, C. Song, S. Jia et al., "An integrated strategy for establishment of curcuminoid profile in turmeric using two LC-MS/MS platforms," Journal of Pharmaceutical and Biomedical Analysis, vol. 132, pp. 93-102, 2017.

[185] K. Inoue, C. Tanada, T. Sakamoto et al., "Metabolomics approach of infant formula for the evaluation of contamination and degradation using hydrophilic interaction liquid chromatography coupled with mass spectrometry," Food Chemistry, vol. 181, pp. 318-324, 2015.

[186] M. Mattarozzi, M. Milioli, F. Bianchi et al., "Optimization of a rapid QuEChERS sample treatment method for HILICMS2 analysis of paralytic shellfish poisoning (PSP) toxins in mussels," Food Control, vol. 60, pp. 138-145, 2016.

[187] A. K. Subbaraj, Y. H. Brad-Kim, K. Fraser, and M. M. Farouk, "A hydrophilic interaction liquid chromatography-mass spectrometry (HILIC-MS) based metabolomics study on colour stability of ovine meat," Meat Science, vol. 117, pp. 163-172, 2016.

[188] G. Aliakbarzadeh, H. Sereshti, and H. Parastar, "Pattern recognition analysis of chromatographic fingerprints of Crocus sativus L. secondary metabolites towards source identification and quality control," Analytical and Bioanalytical Chemistry, vol. 408, no. 12, pp. 3295-3307, 2016.

[189] F. R. Pinu, S. de Carvalho-Silva, A. P. Trovatti Uetanabaro, and S. G. Villas-Boas, "Vinegar metabolomics: an explorative study of commercial balsamic vinegars using gas chromatographymass spectrometry," Metabolites, vol. 6, no. 3, p. 22, 2016.

[190] P. Scano, A. Murgia, F. M. Pirisi, and P. Caboni, "A gas chromatography-mass spectrometry-based metabolomic approach for the characterization of goat milk compared with cow milk," Journal of Dairy Science, vol. 97, no. 10, pp. 6057-6066, 2014.

[191] L. L. Monti, C. A. Bustamante, S. Osorio et al., "Metabolic profiling of a range of peach fruit varieties reveals high metabolic diversity and commonalities and differences during ripening," Food Chemistry, vol. 190, pp. 879-888, 2016.

[192] M. N. A. Khalil, M. I. Fekry, and M. A. Farag, "Metabolome based volatiles profiling in 13 date palm fruit varieties from Egypt via SPME GC-MS and chemometrics," Food Chemistry, vol. 217, pp. 171-181, 2017.

[193] I. Akhatou, R. Gonz alez-Domínguez, and A. Fern andezRecamales, "Investigation of the effect of genotype and agronomic conditions on metabolomic profiles of selected strawberry cultivars with different sensitivity to environmental stress," Plant Physiology and Biochemistry, vol. 101, pp. 14-22, 2016.

[194] A. Cuadros-Ivolstroza, S. Ruíz-Lara, E. Gonz alez, A. Eckardt, L. Willmitzer, and H. Pena-Cortes, "GC-MS metabolic profiling of Cabernet Sauvignon and Merlot cultivars during grapevine berry development and network analysis reveals a stage- and cultivar-dependent connectivity of primary metabolites," Metabolomics, vol. 12, no. 2, p. 39, 2016.

[195] B. Khakimov, R. J. Mongi, K. M. Sørensen, B. K. Ndabikunze, B. E. Chove, and S. B. Engelsen, "A comprehensive and comparative GC-MS metabolomics study of non-volatiles in Tanzanian grown mango, pineapple, jackfruit, baobab and tamarind fruits," Food Chemistry, vol. 213, pp. 691-699, 2016.

[196] J. Welzenbach, C. Neuhoff, C. Looft, K. Schellander, E. Tholen, and C. Große-Brinkhaus, "Different statistical approaches to investigate porcine muscle metabolome profiles to highlight new biomarkers for pork quality assessment," PLoS One, vol. 11, no. 2 article e0149758, 2016.

[197] G. Min-Lee, D. Ho-Suh, E. Sung-Jung, and C. Hwan-Lee, "Metabolomics provides quality characterization of commercial gochujang (fermented pepper paste)," Molecules, vol. 21, no. 7, p. 921, 2016.

[198] D. E. Lee, G. R. Shin, S. Lee et al., "Metabolomics reveal that amino acids are the main contributors to antioxidant activity in wheat and rice gochujangs (Korean fermented red pepper paste)," Food Research International, vol. 87, pp. 10-17, 2016.

[199] E. J. Gu, D. W. Kim, G. J. Jang et al., "Mass-based metabolomic analysis of soybean sprouts during germination," Food Chemistry, vol. 217, pp. 311-319, 2017.

[200] C. Sales, M. I. Cervera, R. Gil, T. Portoles, E. Pitarch, and J. Beltran, "Quality classification of Spanish olive oils by untargeted gas chromatography coupled to hybrid quadrupole-time of flight mass spectrometry with atmospheric pressure chemical ionization and metabolomicsbased statistical approach," Food Chemistry, vol. 216, pp. 365-373, 2017.

[201] D. K. Trivedi, K. A. Hollywood, N. J. W. Rattray et al., "Meat, the metabolites: an integrated metabolite profiling and lipidomics approach for the detection of the adulteration of beef with pork," Analyst, vol. 141, no. 7, pp. 2155-2164, 2016.

[202] V. D. Daygon, S. Prakash, M. Calingacion et al., "Understanding the jasmine phenotype of rice through metabolite profiling and sensory evaluation," Metabolomics, vol. 12, no. 4, p. 63,2016 
[203] L. Di Donna, D. Taverna, S. Indelicato, A. Napoli, G. Sindona, and F. Mazzotti, "Rapid assay of resveratrol in red wine by paper spray tandem mass spectrometry and isotope dilution," Food Chemistry, vol. 229, pp. 354-357, 2017.

[204] H. V. Pereira, V. S. Amador, M. M. Sena, R. Augusti, and E. Piccin, "Paper spray mass spectrometry and PLS-DA improved by variable selection for the forensic discrimination of beers," Analytica Chimica Acta, vol. 940, pp. 104-112, 2016.

[205] J. A. Reis Teodoro, H. V. Pereira, M. M. Sena, E. Piccin, J. J. Zacca, and R. Augusti, "Paper spray mass spectrometry and chemometric tools for a fast and reliable identification of counterfeit blended Scottish whiskies," Food Chemistry, vol. 237, pp. 1058-1064, 2017.

[206] A. K. Meher and Y. C. Chen, "Analysis of volatile compounds by open-air ionization mass spectrometry," Analytica Chimica Acta, vol. 966, pp. 41-46, 2017.

[207] M. Ha, J. H. Kwak, Y. Kim, and O. P. Zee, "Direct analysis for the distribution of toxic glycoalkaloids in potato tuber tissue using matrix-assisted laser desorption/ionization mass spectrometric imaging," Food Chemistry, vol. 133, no. 4, pp. 1155-1162, 2012.

[208] S. Taira, S. Shimma, I. Osaka et al., "Mass spectrometry imaging of the capsaicin localization in the Capsicum fruits," International Journal of Biotechnology for Wellness Industries, vol. 1, pp. 61-66, 2012.

[209] Y. Yoshimura, H. Evolmoto, T. Moriyama, Y. Kawamura, M. Setou, and N. Zaima, "Visualization of anthocyanin species in rabbiteye blueberry Vaccinium ashei by matrixassisted laser desorption/ionization imaging mass spectrometry," Analytical and Bioanalytical Chemistry, vol. 403, no. 7, pp. 885-1895, 2012.

[210] A. Yasuda, Y. Tatsu, and Y. Shigeri, "Characterization of triacetyl- $\alpha$-melanocyte-stimulating hormone in carp and goldfish," General and Comparative Endocrinology, vol. 175, no. 2, pp. 270-276, 2012.

[211] M.-Z. Huang, S.-C. Cheng, S.-S. Jhang et al., “Ambient molecular imaging of dry fungus surface by electrospray laser desorption ionization mass spectrometry," International Journal of Mass Spectrometry, vol. 325-327, pp. 172-182, 2012.

[212] S. S. Jhang, M.-Z. Huang, and J. Shiea, “Ambient molecular imaging of toxins within a sprouted potato slice by ELDI/MS," in Proceedings of the 60th ASMS Conference on Mass Spectrometry and Allied Topics, Vancouver, BC, Canada, May 2012.

[213] R. Garrett, C. M. Rezende, and D. R. Ifa, "Revealing the spatial distribution of chlorogenic acids and sucrose across coffee bean endosperm by desorption electrospray ionization-mass spectrometry imaging," LWT-Food Science and Technology, vol. 65, pp. 711-717, 2016.

[214] P. M. Kumara, A. Srimany, G. Ravikanth, R. U. Shaanker, and T. Pradeep, "Ambient ionization mass spectrometry imaging of rohitukine, a chromone anti-cancer alkaloid, during seed development in Dysoxylum binectariferum Hook.f (Meliaceae)," Phytochemistry, vol. 116, pp. 104-110, 2015.

[215] M. W. Nielen and T. A. van Beek, "Macroscopic and microscopic spatially-resolved analysis of food contaminants and constituents using laser-ablation electrospray ionization mass spectrometry imaging," Analytical and Bioanalytical Chemistry, vol. 406, no. 27, pp. 6805-6815, 2014. 

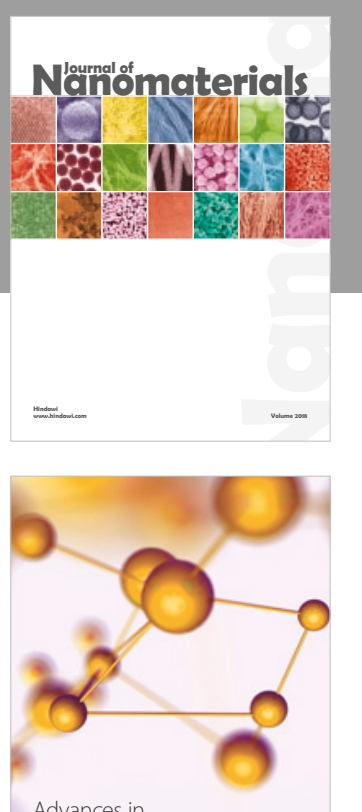

Physical Chemistry
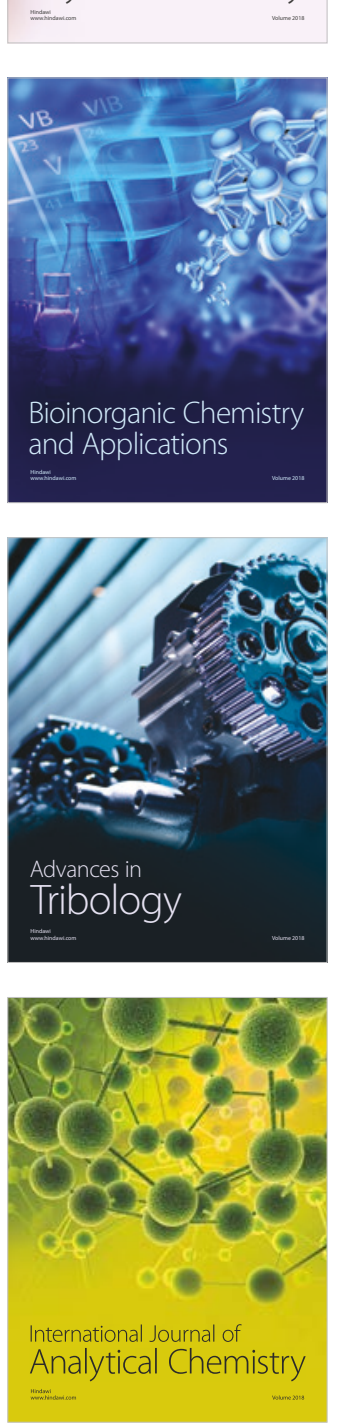

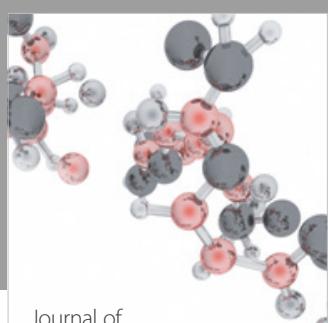

Analytical Methods

in Chemistry

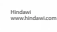

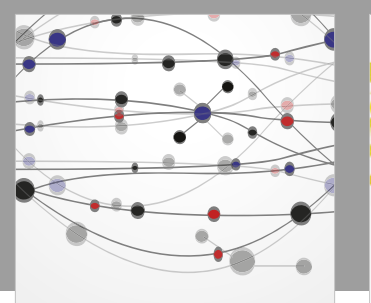

The Scientific World Journal

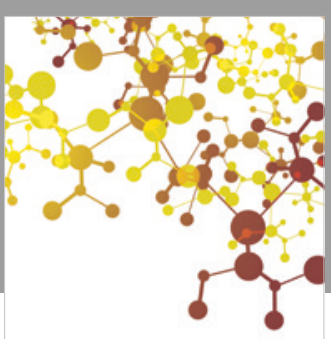

Journal of

Applied Chemistry
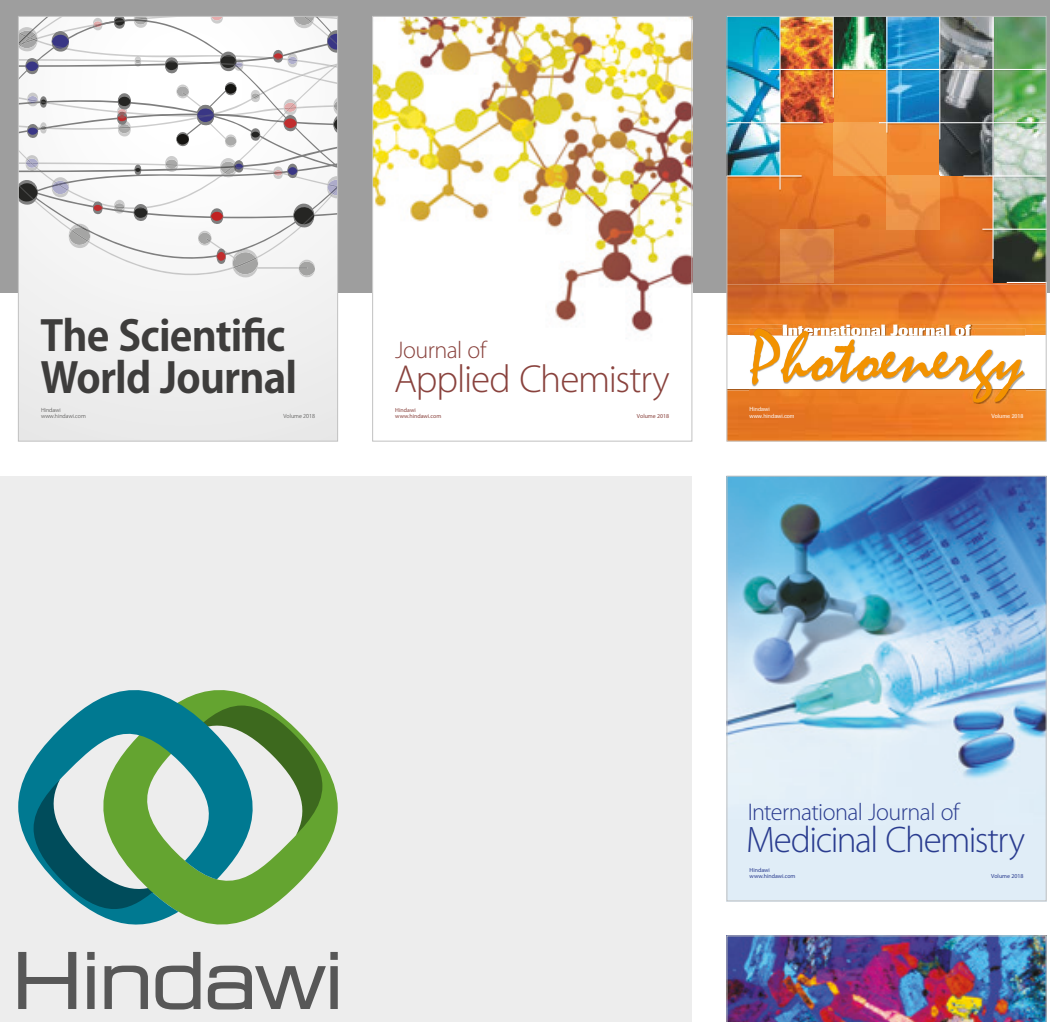

Submit your manuscripts at

www.hindawi.com
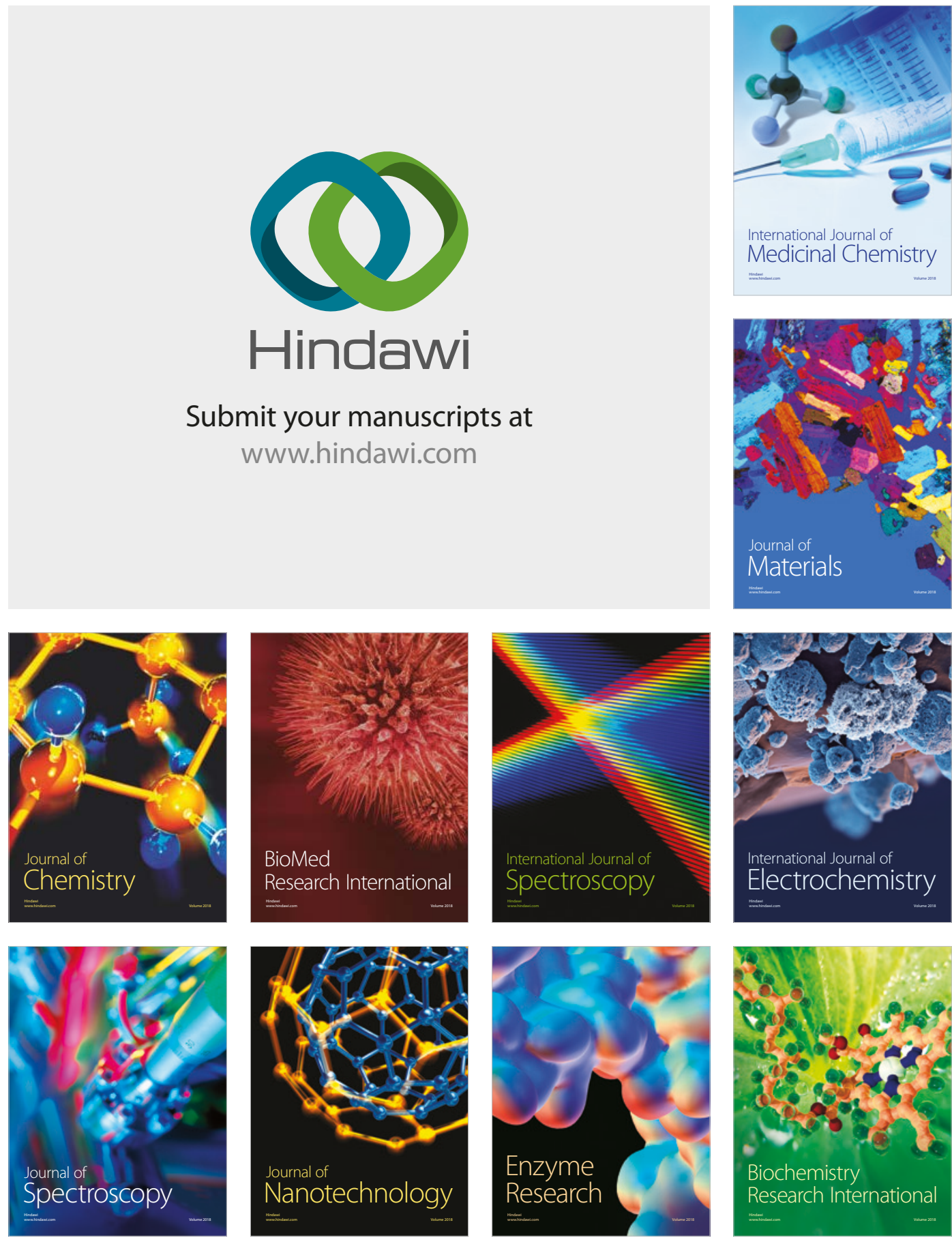
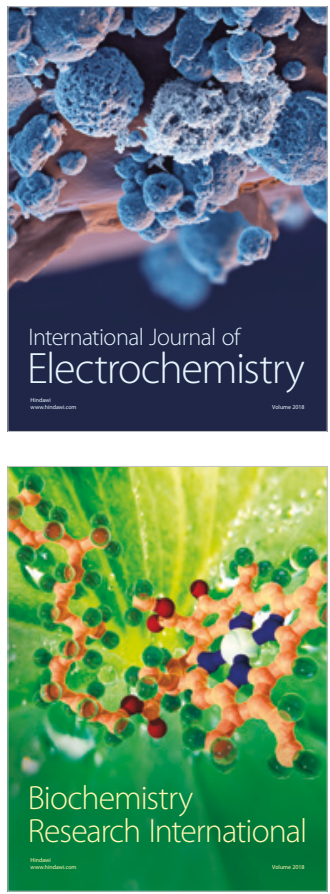